\title{
Three-way group decisions with decision-theoretic rough sets
}

\author{
Decui Liang ${ }^{\mathrm{a}, *}$, Dun Liu ${ }^{\mathrm{b}}$, Agbodah Kobina ${ }^{\mathrm{a}}$ \\ ${ }^{a}$ School of Management and Economics, University of Electronic Science and Technology of China, Chengdu 610054, China \\ ${ }^{b}$ School of Economics and Management, Southwest Jiaotong University, Chengdu 610031, China
}

\begin{abstract}
On consideration of the effectiveness of group decision making (GDM) in practical complex decision problems, we introduce GDM into three-way decisions with decision-theoretic rough sets (DTRSs) and propose GDM-based three-way decisions. GDM-based three-way decisions extend the range of applications of three-way decisions with DTRSs and provide a novel interpretation of the determination of loss functions. Based on DTRSs, we firstly focus on analyzing the determination for the loss functions under the GDM environment. With the aid of the principle of justifiable granularity, we adopt the important and majority suggestions of experts to measure each loss function, which supports a coherent way of designing information granules in presence of numerics. In this case, the loss functions are determined in the form of interval-valued information granule. By using the interval comparison method, we further deduce the three-way decisions and design a corresponding decision procedure of GDM-based three-way decisions. Then, an example of strategy supply selection is given to elaborate the GDM-based three-way decisions. Finally, we validate the performance of our proposed method by experimental analysis.
\end{abstract}

Keywords: Three-way decisions, Decision-theoretic rough sets, Group decision making, Granular computing, Interval analysis

\section{Introduction}

Three-way decisions are commonly encountered in problem solving strategies $[14,48,50]$. As a novel method, it has been applied in many fields, such as ranking and resource allocation [22], investment decisions [20, 27], information filtering [16], text classification [17], risk decisions [18, 22], cluster analysis [25, 52, 54], government decisions [29], web-based support systems [45, 47], recommender systems [56], email spam filtering [59], etc. The decisions of acceptance, rejection and non-commitment are the general interpretations of three-way decisions. On the basis of different decision problems, we need to explain the associated semantics of three-way decisions, respectively. For instance, in medical decision making, they may be interpreted as treatment, no treatment and further testing. The idea of three-way decisions is consistent with human's cognitions to solve the problem in the real world. With respect to the research content of three-way decisions, decision-theoretic rough sets (DTRSs) are typical models of three-way decisions [7, 19, 31, 33, 48, 50, 53, 57, 58]. DTRSs were proposed by Yao et al. [48, 49] in the framework of the Bayesian decision procedure [8] and rough set theory [34]. It not only considers decision risk factors, but also involves three types of decision actions, i.e., the acceptance, the non-commitment and the rejection [28, 32, 40]. Three-way decisions with DTRSs are derived from the minimum of the risk of decision making. As an extension model of rough sets [34], DTRSs consider the practical semantics and vastly push the development of three-way decisions. For example, on basis of the costs of misclassification, Greco et al. [12] proposed a new dominance-based rough set approach. In the viewpoint of the optimization, Jia et al. [15] discussed the determination of the thresholds and the reduction. Qian et al. [39] developed a multi-granulation DTRSs.

For three-way decisions with DTRSs, the determination of loss function is a pivotal step in the decision procedure [22]. For example, Li and Zhou [18] discussed the determination of loss functions based on different attitudes of

\footnotetext{
*corresponding author.

Email addresses: decuiliang@126.com (Decui Liang), newton83@163.com (Dun Liu), kobinaagbodah@yahoo.com (Agbodah Kobina)
} 
decision makers. Lingras et al. [25] redefined a loss function using the rough clusters. By introducing game theory into probabilistic rough sets, Yao and Herbert [46] proposed game-theoretic rough sets (GTRSs) to determine the values of thresholds. With regard to the loss function of DTRSs, Yu et al. [52] constructed a new clustering validity evaluation function. Liu et al. [27] used the profit function to measure the loss function for the investment decisionmaking. During the calculation procedure, Yao [50] employed relative values to estimate loss functions. Liang et al. [20], Liang and Liu [21, 22, 23, 24] and Liu et al. [30] effectively estimated the loss function with the aid of fuzzy numbers and intervals. Considering the preferences of decision makers, Yang and Yao [44] discussed some aggregations of the thresholds. They extend the range of applications of three-way decisions with DTRSs.

With the aforementioned literatures, the determination of loss function of DTRSs is associated with decision makers. The loss functions in the most of existing studies are evaluated by single decision maker. In the face of complicated decision environment, the single decision maker may be difficult to make a reasonable decision [20, 43, 44], e.g., limited domain knowledge of decision maker, tight deadlines, limited budgets [20]. Fortunately, group decision making (GDM) provides an effective evaluation approach [30], which can aggregate the wisdom of the different domain experts and effectively cope with risk decision problems [20, 41, 43]. For example, at a plan moment of new production development, the development decision not only depends on the senior management, but also should rely on the opinions of the engineers and salesmen. GDM also exists in our daily life, e.g., the strategic supplier selection [3], the public-private partnership (PPP) project investment [20], etc. However, we may face the inconsistency among experts under the GDM environment [43]. For example, the score of one student is evaluated by four experts. The results are 55, 82, 85 and 90. Obviously, there is a large difference between 55 and 90 . Reaching consensus in GDM becomes an essential step [20]. The consensus among experts reflects their consistent opinions. Generally speaking, the combination of GDM and three-way decisions has not received sufficient research attentions. The loss functions of DTRSs evaluated by GDM need to be deeply investigated.

Considering the effectiveness of GDM in practical complex decision problems, we explore novel three-way decisions with DTRSs. Under the GDM environment, we mainly discuss the determination of loss function and deduce three-way decisions. GDM provides a novel semantic interpretation for the loss functions of three-way decisions. Meanwhile, it also may bring the inconsistency among the opinions of multiple experts [20]. Based on a group of numerics, Pedrycz [38] systematically discussed the building of information granules with the aid of the principle of justifiable granularity, which can abstract the meaning of numerics. The principle of justifiable granularity is a new approach to aggregate the group opinion of multiple experts [36, 37, 42]. It can get the important and majority suggestions of experts and improve the evaluation effectiveness of GDM. The interval format is a typical information granule [21]. Thus, we adopt the principle of justifiable granularity to abstract the experts' evaluation results and determine the loss functions with intervals. By utilizing a suitable interval analysis method, we further derive three-way decisions. Then, the decision procedure of GDM-based three-way decisions is designed. Taking the strategy supply selection as an example, we illustrate the GDM-based three-way decisions. Comparing with the results of Yang and Yao [44], we focus on the determination of loss functions and involve the inconsistency among the opinions of multiple experts. The main contributions of our work can be identified as follows: (1) We extend the range of applications of three-way decisions to GDM and provide a novel interpretation of the determination of loss functions ; (2) The principle of justifiable granularity explains the imprecise origin of the existing literatures, e.g., Liang et al. [20], Liang and Liu $[21,22,23]$.

The remainder of this paper is organized as follows. Section 2 lists some symbols used in this paper in advance. Section 3 provides basic concepts of three-way decisions with DTRSs. Under the GDM environment, GDM-based three-way decisions are proposed in Section 4. Then, a case study of strategy supply selection is given to illustrate our proposed method in Section 5. In Section 6, we further validate the performance of our proposed method by experimental analysis. Section 7 concludes the paper and outlines the future work.

\section{Notations}

Under the GDM environment, we extend three-way decisions with DTRSs to a general situation and propose GDM-based three-way decisions. Many symbols are involved in this study and for clarity most of them are summarized in Table 1. 
Table 1: List of symbols

\begin{tabular}{|c|c|}
\hline Symbol & Description \\
\hline$x$ & An object \\
\hline$\Omega=\{C, \neg C\}$ & The set of states, indicating that an object is in $C$ and not in $C$ \\
\hline$P$ & The object is in $C$ \\
\hline$N$ & The object is not in $C$ \\
\hline $\operatorname{POS}(C)$ & The acceptance decision of $x \in C$ \\
\hline $\mathrm{BND}(C)$ & The deferment (non-commitment) decision of $x \in C$ \\
\hline $\operatorname{NEG}(C)$ & The rejection decision of $x \in C$ \\
\hline $\mathcal{A}=\left\{a_{P}, a_{B}, a_{N}\right\}$ & $\begin{array}{l}\text { The set of actions, where } a_{P}, a_{B} \text {, and } a_{N} \text { represent three actions when classifying object } x \text {, namely, } \\
\text { deciding } x \in \operatorname{POS}(C) \text {, deciding } x \text { should be further investigated } x \in \operatorname{BND}(C) \text {, and deciding } x \in \operatorname{NEG}(C)\end{array}$ \\
\hline$\Delta$ & The symbol of action, e.g., $\triangle=P, B, N$ \\
\hline$\nabla$ & The symbol of state, e.g., $\nabla=P, N$ \\
\hline$\lambda_{\triangle P}$ & The loss incurred for taking actions of $a_{\Delta}$ when an object belongs to $C$ \\
\hline$\lambda_{\Delta N}$ & The loss incurred for taking actions of $a_{\triangle}$ when an object belongs to $\neg C$ \\
\hline $\operatorname{Pr}(C \mid[x])$ & The conditional probability of an object $x$ belonging to $C$ \\
\hline $\operatorname{Pr}(\neg C \mid[x])$ & The conditional probability of an object $x$ belonging to $\neg C$ \\
\hline$R\left(a_{\Delta} \mid[x]\right)$ & The expected loss associated with taking the individual action $a_{\Delta}$ \\
\hline$\alpha, \beta, \gamma$ & The threshold values \\
\hline$t$ & The number of experts \\
\hline$t^{\prime}$ & The number of different values shown in the loss function evaluation of experts \\
\hline$E=\left\{e_{1}, e_{2}, \cdots, e_{t}\right\}$ & The set of experts \\
\hline$W=\left\{w_{1}, w_{2}, \cdots, w_{t}\right\}^{T}$ & The weight vector of experts \\
\hline$\lambda_{\Delta \nabla}^{k}$ & The value of loss function $\lambda_{\Delta \nabla}$ evaluated by the expert $e_{k}(1 \leq k \leq t)$ \\
\hline$\lambda_{\Delta \nabla}^{\sigma(k)}$ & The $k$ th value of the evaluations of all experts for $\lambda_{\Delta \nabla}$ in an ascending order $(1 \leq k \leq t)$ \\
\hline$w_{\Delta \nabla}^{\sigma(k)}$ & The corresponding weight information for the $k$ th value of the evaluations of all experts \\
\hline$\lambda_{\Delta \nabla}^{-}$ & The lower bound for the loss function $\lambda_{\Delta \nabla}$ \\
\hline$\lambda_{\Delta \nabla}^{+}$ & The upper bound for the loss function $\lambda_{\Delta \nabla}$ \\
\hline$V\left(l_{\Delta \nabla}\right)$ & The optimization function for determining the lower bound of the loss function $\lambda_{\Delta \nabla}$ \\
\hline$V\left(u_{\Delta \nabla}\right)$ & The optimization function for determining the upper bound of the loss function $\lambda_{\Delta \nabla}$ \\
\hline$m_{\Delta \nabla}$ & A weighted mean of $\lambda_{\Delta \nabla}$ \\
\hline$\sigma(N(y))$ & The location of the value $y$ presented in the evaluations of loss function $\lambda_{\Delta \nabla}$ according to an ascending order \\
\hline$\varepsilon$ & A positive parameter offering some flexibility for the produced information granule \\
\hline$d\left(\lambda_{\Delta \nabla}\right)_{1}$ & The inconsistency of GDM with the average deviation method \\
\hline$d\left(\lambda_{\Delta \nabla}\right)_{2}$ & The inconsistency of GDM with the principle of justifiable granularity \\
\hline$\theta$ & The risk attitude parameter of decision makers \\
\hline$m_{\theta}(\lambda)$ & The transformed formula of an interval $\lambda=\left[\lambda^{-}, \lambda^{+}\right]$, which is changed into a real number \\
\hline$e$ & The error rate \\
\hline$Q$ & The total number of objects presented in the universe \\
\hline$n_{C \rightarrow \mathrm{NEG}(C)}$ & The number of objects belonging to $C$ that are classified as belonging to $\mathrm{NEG}(C)$ \\
\hline$n_{\neg C \rightarrow \mathrm{POS}(C)}$ & The number of objects belonging to $\neg C$ that are classified as belonging to $\operatorname{POS}(C)$ \\
\hline
\end{tabular}


Table 2: The loss function regarding the risk or cost of actions in different states

\begin{tabular}{|l|c|c|}
\hline & $C(P)$ & $\neg C(N)$ \\
\hline$a_{P}$ & $\lambda_{P P}$ & $\lambda_{P N}$ \\
$a_{B}$ & $\lambda_{B P}$ & $\lambda_{B N}$ \\
$a_{N}$ & $\lambda_{N P}$ & $\lambda_{N N}$ \\
\hline
\end{tabular}

\section{A brief review of three-way decisions with decision-theoretic rough sets (DTRSs)}

In this section, we briefly review the three-way decisions derived from DTRSs. In light of Bayesian decision procedure [8] and rough set theory [34], Yao [48, 49, 50] assumed that DTRSs were composed of two states and three actions. With respect to the relevant concepts of rough sets, the set of states is given by $\Omega=\{C, \neg C\}$ indicating that an object is in $C$ and not in $C$, respectively. In the context of different decision making problems, we define their own meanings of the states. The set of actions is given by $\mathcal{A}=\left\{a_{P}, a_{B}, a_{N}\right\}$, where $a_{P}, a_{B}$, and $a_{N}$ represent three actions when classifying object $x$, namely, deciding $x \in \operatorname{POS}(C)$, deciding $x$ should be further investigated $x \in \operatorname{BND}(C)$, and deciding $x \in \operatorname{NEG}(C)$, respectively. At this point, $\operatorname{POS}(C), \operatorname{BND}(C)$ and $\operatorname{NEG}(C)$ comprise three-way decisions, which correspond to the acceptance decision, the deferment (non-commitment) decision and the rejection decision $[50,51,55]$. Our final decisions rely on the set of actions. The loss function regarding the risk or cost of actions in the different states is given by a $3 \times 2$ matrix in Table 2 .

From the result presented in Table 2, six types of loss functions are associated with our evaluations: $\lambda_{P P}, \lambda_{B P}, \lambda_{N P}$, $\lambda_{P N}, \lambda_{B N}$ and $\lambda_{N N}$. More specifically, $\lambda_{P P}, \lambda_{B P}$ and $\lambda_{N P}$ denote the losses incurred for taking actions of $a_{P}, a_{B}$ and $a_{N}$, respectively, when an object belongs to $C$. Similarly, $\lambda_{P N}, \lambda_{B N}$ and $\lambda_{N N}$ denote the losses incurred for taking the same actions when the object belongs to $\neg C$. By considering a reasonable kind of loss functions with [49]

$$
\begin{aligned}
& \lambda_{P P} \leq \lambda_{B P}<\lambda_{N P}, \\
& \lambda_{N N} \leq \lambda_{B N}<\lambda_{P N} .
\end{aligned}
$$

where (1) and (2) are the prerequisites imposed on loss functions of DTRSs. As an example of medical diagnosis [22], we explain the relationship among $\lambda_{P P}, \lambda_{B P}$ and $\lambda_{N P} . \lambda_{N P}$ represents the loss of not treating a flu patient, and $\lambda_{P P}$ denotes the loss of treating a flu patient. For a flu patient, not treating may worsen medical conditions or cause death. Hence, the loss of classifying an object $x$ belonging to $C$ into the positive region POS $(C)$ is less than or equal to the loss of classifying $x$ into the boundary region $\operatorname{BND}(C)$, and both of these losses are strictly less than the loss of classifying $x$ into the negative region $\operatorname{NEG}(C)$. The reverse orders of these loss functions (i.e., $\lambda_{N N}, \lambda_{B N}$ and $\lambda_{P N}$ ) are suitable for classifying an object not in $C$. Let $\operatorname{Pr}(C \mid[x])$ be the conditional probability of an object $x$ belonging to $C$ given that the object is described by its equivalence class $[x]$. Analogously, $\operatorname{Pr}(\neg C \mid[x])$ is the conditional probability of an object $x$ belonging to $\neg C$ and $\operatorname{Pr}(C \mid[x])+\operatorname{Pr}(\neg C \mid[x])=1$.

For an object $x$, the expected losses associated with taking the individual action can be expressed as:

$$
\begin{aligned}
& R\left(a_{P} \mid[x]\right)=\lambda_{P P} \operatorname{Pr}(C \mid[x])+\lambda_{P N} \operatorname{Pr}(\neg C \mid[x]), \\
& R\left(a_{B} \mid[x]\right)=\lambda_{B P} \operatorname{Pr}(C \mid[x])+\lambda_{B N} \operatorname{Pr}(\neg C \mid[x]), \\
& R\left(a_{N} \mid[x]\right)=\lambda_{N P} \operatorname{Pr}(C \mid[x])+\lambda_{N N} \operatorname{Pr}(\neg C \mid[x]) .
\end{aligned}
$$

The Bayesian decision procedure implies the following minimum-cost decision rules:

(P) If $R\left(a_{P} \mid[x]\right) \leq R\left(a_{B} \mid[x]\right)$ and $R\left(a_{P} \mid[x]\right) \leq R\left(a_{N} \mid[x]\right)$, decide $x \in \operatorname{POS}(C)$;

(B) If $R\left(a_{B} \mid[x]\right) \leq R\left(a_{P} \mid[x]\right)$ and $R\left(a_{B} \mid[x]\right) \leq R\left(a_{N} \mid[x]\right)$, decide $x \in \operatorname{BND}(C)$;

(N) If $R\left(a_{N} \mid[x]\right) \leq R\left(a_{P} \mid[x]\right)$ and $R\left(a_{N} \mid[x]\right) \leq R\left(a_{B} \mid[x]\right)$, decide $x \in \operatorname{NEG}(C)$.

where decision rules $(\mathrm{P})-(\mathrm{N})$ are the three-way decisions proposed by Yao [50, 51]. The three-way decisions comprise the positive rules $(\mathrm{P})$, the boundary rules $(\mathrm{B})$, and the negative rules $(\mathrm{N})$. The positive rules make decisions of acceptance. The negative rules make decisions of rejection, and the boundary rules make decisions of non-commitment. 
Table 3: The evaluation results of loss functions of the expert $e_{k}$

\begin{tabular}{|l|c|c|}
\hline$e_{k}$ & $C(P)$ & $\neg C(N)$ \\
\hline$a_{P}$ & $\lambda_{P P}^{k}$ & $\lambda_{P N}^{k}$ \\
$a_{B}$ & $\lambda_{B P}^{k}$ & $\lambda_{B N}^{k}$ \\
$a_{N}$ & $\lambda_{N P}^{k}$ & $\lambda_{N N}^{k}$ \\
\hline
\end{tabular}

Since $\operatorname{Pr}(C \mid[x])+\operatorname{Pr}(\neg C \mid[x])=1$, we simplify the decision rules based only on the probability $\operatorname{Pr}(C \mid[x])$ and the loss functions. The decision rules $(\mathrm{P})-(\mathrm{N})$ can be expressed concisely as follows:

(P) $\quad$ If $\operatorname{Pr}(C \mid[x]) \geq \alpha$ and $\operatorname{Pr}(C \mid[x]) \geq \gamma$, decide $x \in \operatorname{POS}(C)$;

(B) $\quad$ If $\operatorname{Pr}(C \mid[x]) \leq \alpha$ and $\operatorname{Pr}(C \mid[x]) \geq \beta$, decide $x \in \operatorname{BND}(C)$;

(N) $\quad$ If $\operatorname{Pr}(C \mid[x]) \leq \beta$ and $\operatorname{Pr}(C \mid[x]) \leq \gamma$, decide $x \in \mathrm{NEG}(C)$.

The threshold values $\alpha, \beta, \gamma$ are given by:

$$
\begin{aligned}
\alpha & =\frac{\left(\lambda_{P N}-\lambda_{B N}\right)}{\left(\lambda_{P N}-\lambda_{B N}\right)+\left(\lambda_{B P}-\lambda_{P P}\right)}, \\
\beta & =\frac{\left(\lambda_{B N}-\lambda_{N N}\right)}{\left(\lambda_{B N}-\lambda_{N N}\right)+\left(\lambda_{N P}-\lambda_{B P}\right)}, \\
\gamma & =\frac{\left(\lambda_{P N}-\lambda_{N N}\right)}{\left(\lambda_{P N}-\lambda_{N N}\right)+\left(\lambda_{N P}-\lambda_{P P}\right)} .
\end{aligned}
$$

From decision rules $(\mathrm{P})-(\mathrm{N})$, the decision result of the object $x$ can be obtained by comparing the conditional probability $\operatorname{Pr}(C \mid[x])$ and the thresholds $(\alpha, \beta, \gamma)$. The detailed derivation process refers to the results presented in $[48,49]$. In addition, Yao [50, 51] further simplified $(\mathrm{P})-(\mathrm{N})$ under some specific conditions of loss functions.

\section{Group decision making (GDM)-based three-way decisions}

As stated in Section 3, three-way decisions with DTRSs mainly focus on the single decision-maker's evaluation. Based on DTRSs and the results reported in [20], this section extends the single decision making situation to GDM. Under the GDM context, we discuss the determination of loss function and propose GDM-based three-way decisions.

\subsection{Basic notations of GDM-based three-way decisions}

Following the notations presented in Section 3, the classification problem considers two states $\Omega=\{C, \neg C\}$ and three actions $\mathcal{A}=\left\{a_{P}, a_{B}, a_{N}\right\}$. During the realistic evaluation process, $t$ experts are invited to evaluate the loss functions of Table $2(t \geq 3)$. Let $E=\left\{e_{1}, e_{2}, \cdots, e_{t}\right\}$ be the set of experts and $W=\left\{w_{1}, w_{2}, \cdots, w_{t}\right\}^{T}$ be the weight vector of experts, where $\sum_{k=1}^{t} w_{k}=1$ and $w_{k} \geq 0$. For convenience, we focus on the determination of loss functions and assume the weights of the experts are given. According to the loss functions of Table 2 and (1)-(2), each expert assigns the values of loss functions. The evaluation results of the expert $e_{k}$ are shown in Table $3(1 \leq k \leq t)$.

From the results of Table 3, the values of loss functions regarding the risk or cost of actions in the different states are $\lambda_{P P}^{k}, \lambda_{B P}^{k}, \lambda_{N P}^{k}, \lambda_{P N}^{k}, \lambda_{B N}^{k}$ and $\lambda_{N N}^{k}$, i.e., the value of loss function $\lambda_{\Delta \nabla}$ evaluated by the expert $e_{k}$ is $\lambda_{\Delta \nabla}^{k}(\Delta=$ $P, B, N ; \nabla=P, N)$. The evaluation results of all experts are summarized in Table 4 .

For the evaluations of each expert, the values of loss functions in Table 4 obey the following relationships: $\lambda_{P P}^{k} \leq$ $\lambda_{B P}^{k}<\lambda_{N P}^{k}$ and $\lambda_{N N}^{k} \leq \lambda_{B N}^{k}<\lambda_{P N}^{k}(1 \leq k \leq t)$. 
Table 4: The evaluation results of all experts

\begin{tabular}{|c|c|c|c|c|c|c|}
\hline$E$ & $\lambda_{P P}$ & $\lambda_{B P}$ & $\lambda_{N P}$ & $\lambda_{P N}$ & $\lambda_{B N}$ & $\lambda_{N N}$ \\
\hline$e_{1}$ & $\lambda_{P P}^{1}$ & $\lambda_{B P}^{1}$ & $\lambda_{N P}^{1}$ & $\lambda_{P N}^{1}$ & $\lambda_{B N}^{1}$ & $\lambda_{N N}^{1}$ \\
$e_{2}$ & $\lambda_{P P}^{2}$ & $\lambda_{B P}^{2}$ & $\lambda_{N P}^{2}$ & $\lambda_{P N}^{2}$ & $\lambda_{B N}^{2}$ & $\lambda_{N N}^{2}$ \\
$\vdots$ & $\vdots$ & $\vdots$ & $\vdots$ & $\vdots$ & $\vdots$ & $\vdots$ \\
$e_{k}$ & $\lambda_{P P}^{k}$ & $\lambda_{B P}^{k}$ & $\lambda_{N P}^{k}$ & $\lambda_{P N}^{k}$ & $\lambda_{B N}^{k}$ & $\lambda_{N N}^{k}$ \\
$\vdots$ & $\vdots$ & $\vdots$ & $\vdots$ & $\vdots$ & $\vdots$ & $\vdots$ \\
$e_{t}$ & $\lambda_{P P}^{t}$ & $\lambda_{B P}^{t}$ & $\lambda_{N P}^{t}$ & $\lambda_{P N}^{t}$ & $\lambda_{B N}^{t}$ & $\lambda_{N N}^{t}$ \\
\hline
\end{tabular}

\subsection{The determination of loss function with the principle of justifiable granularity}

In Table 4, each loss function presented in Table 2 has multiple evaluation results in the GDM. Taking the loss function $\lambda_{P P}$ as an example, there are $\lambda_{P P}^{1}, \lambda_{P P}^{2}, \cdots$, and $\lambda_{P P}^{t}$. In this stage, we need to aggregate the evaluation information of all experts for determining the loss functions. The purpose is to obtain the important and majority suggestions of experts and improve the evaluation effectiveness of GDM. More specifically, it needs to abstract the characteristics from the data set and improve the inconsistency of GDM [20, 43]. Fortunately, Pedrycz [38] proposed a principle of justifiable granularity, which was concerned with a formation of a meaningful representation of a collection of numerics. By using the principle of justifiable granularity, Pedrycz and Song [35] discussed a construction of interval-based information granules in the present of some numeric evidences. Wang et al. [42] studied the partition of the universe of discourse into intervals with the principle of justifiable granularity to improve forecasting quality. Cabrerizo et al. [4] applied this method into a reciprocal GDM defined in heterogeneous contexts. The principle of justifiable granularity satisfies our requirements, which can abstract the characteristics from the data set and improve the inconsistency of GDM. It supports a coherent way of designing information granules in presence of experimental evidence (either of numerical or granular character) [37]. Therefore, the principle of justifiable granularity is a suitable approach for aggregating the loss function and also can be used to determine the values of loss functions.

According to the results of [38], the schema of the information granulation of loss function under GDM situation is shown in Fig. 1.

In light of the schema presented in Fig. 1, we utilize the principle of justifiable granularity to realize the information granulation of each loss function with an interval format. With regards to the loss function $\lambda_{\Delta \nabla}$, the evaluation results of all experts are obtained as follows: $\lambda_{\Delta \nabla}^{1}, \lambda_{\Delta \nabla}^{2}, \cdots, \lambda_{\Delta \nabla}^{k}, \cdots, \lambda_{\Delta \nabla}^{t}(1 \leq k \leq t)$. Considering the weights of experts, the values of loss function attach a set of weight, namely, $w_{1}, w_{2}, \cdots, w_{k} \cdots, w_{t}$. For the evaluation results of $\lambda_{\Delta \nabla}$, we merge the same values and sort them in an ascending order. The result is:

$$
\lambda_{\Delta \nabla}^{\sigma(1)}, \lambda_{\Delta \nabla}^{\sigma(2)}, \cdots, \lambda_{\Delta \nabla}^{\sigma(k)}, \cdots, \lambda_{\Delta \nabla}^{\sigma\left(t^{\prime}\right)} .
$$

where $\lambda_{\Delta \nabla}^{\sigma(k)}$ denotes the $k$ th value of all experts' evaluations and $1 \leq k \leq t^{\prime} \leq t$. Meanwhile, we obtain their corresponding weight information:

$$
w_{\Delta \nabla}^{\sigma(1)}, w_{\Delta \nabla}^{\sigma(2)}, \cdots, w_{\Delta \nabla}^{\sigma(k)}, \cdots, w_{\Delta \nabla}^{\sigma\left(t^{\prime}\right)} .
$$

In order to obtain the important and majority suggestions of experts, we granulate the evaluations and determine its lower and upper bound for the loss function $\lambda_{\Delta \nabla}$. We are concerned with the realization of interval information granule based on the outcomes of the evaluations, which is regarded as an optimization problem [38, 42]. The optimized interval of $\lambda_{\Delta \nabla}$ is denoted as $\lambda_{\Delta \nabla}=\left[\lambda_{\Delta \nabla}^{-}, \lambda_{\Delta \nabla}^{+}\right] . \lambda_{\Delta \nabla}^{-}$and $\lambda_{\Delta \nabla}^{+}$denote the lower and upper bound of the loss function $\lambda_{\Delta \nabla}$, respectively. On the basis of the results reported in [38, 42], we consider a maximization of the composite multiplicative index that is realized independently for the lower and upper bound of an interval:

$$
V\left(l_{\Delta \nabla}\right)=\exp \left(-\varepsilon\left|m_{\Delta \nabla}-l_{\Delta \nabla}\right|\right) * \sum_{l_{\Delta \nabla} \leq y \leq m_{\Delta \nabla}} w_{\Delta \nabla}^{\sigma(N(y))}
$$




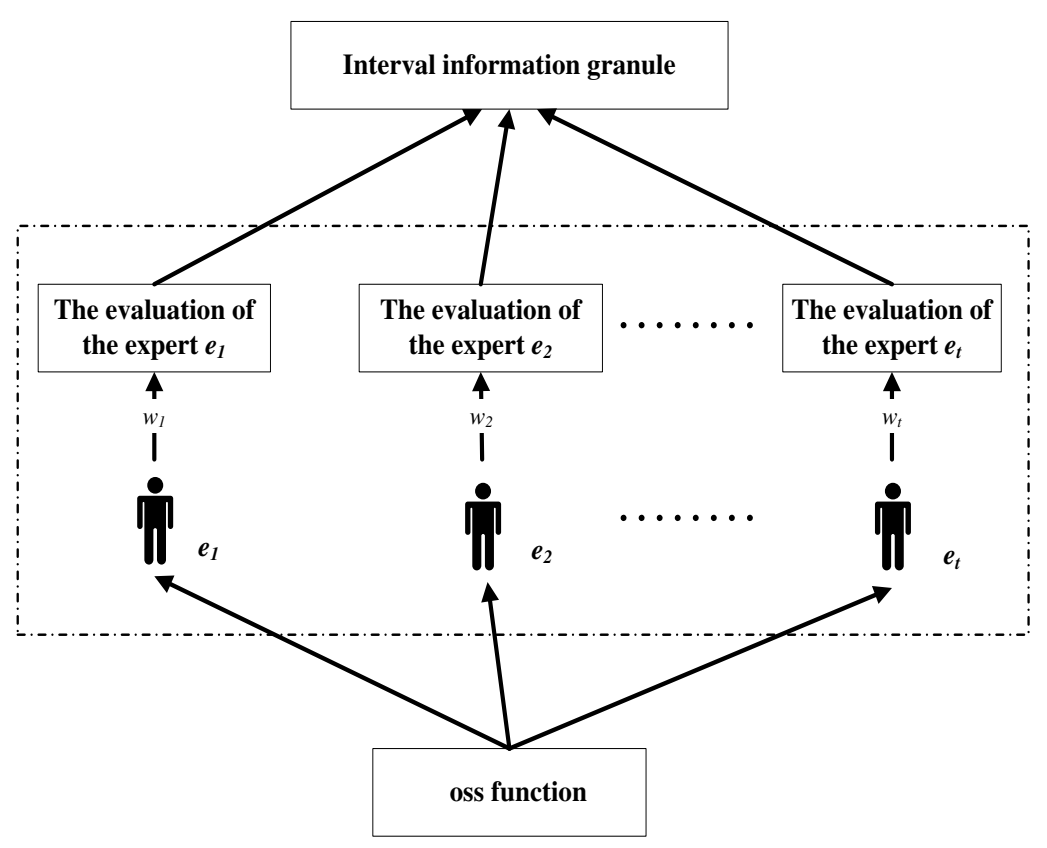

Figure 1: The schema of the information granulation of loss function

$$
V\left(u_{\Delta \nabla}\right)=\exp \left(-\varepsilon\left|m_{\Delta \nabla}-u_{\Delta \nabla}\right|\right) * \sum_{m_{\Delta \nabla} \leq y \leq u_{\Delta \nabla}} w_{\Delta \nabla}^{\sigma(N(y))} .
$$

where $l_{\Delta \nabla}$ and $u_{\Delta \nabla}$ stand for the lower and upper bound, respectively. $V\left(l_{\Delta \nabla}\right)$ and $V\left(u_{\Delta \nabla}\right)$ are the optimization functions and $l_{\Delta \nabla} \leq u_{\Delta \nabla} . \varepsilon$ is a positive parameter offering some flexibility for the produced information granule. The essential role of $\varepsilon$ is to calibrate the constructed information granule [37]. $\sigma(N(y))$ is a position of the value $y$ presented in the evaluations of loss function $\lambda_{\Delta \nabla}$ according to an ascending order. At this point, $m_{\Delta \nabla}$ is a weighted mean of $\lambda_{\Delta \nabla}$. Due to the weights of the experts [20,43], we normally use the mean to describe the group decision result. It is calculated as:

$$
m_{\Delta \nabla}=\sum_{k=1}^{t} w_{\Delta \nabla}^{\sigma(k)} \lambda_{\Delta \nabla}^{\sigma(k)}
$$

Under the GDM environment, the relationship among the weighted means of loss functions $\lambda_{\Delta \nabla}$ presented in Table 4 is captured as follows.

Proposition 1. Based on (1) and (2), the weighted means of loss functions presented in Table 4 imply the following relationship: $m_{P P} \leq m_{B P}<m_{N P}$ and $m_{N N} \leq m_{B N}<m_{P N}$.

Proof. With regards to each loss function $\lambda_{\Delta \nabla}$, the evaluation results of all experts are obtained as follows: $\lambda_{\Delta \nabla}^{1}$, $\lambda_{\Delta \nabla}^{2}, \cdots, \lambda_{\Delta \nabla}^{k}, \cdots, \lambda_{\Delta \nabla}^{t}(\triangle=P, B, N ; \nabla=P, N ; 1 \leq k \leq t)$. Comparing $\lambda_{P P}$ with $\lambda_{B P}$, we have: $\lambda_{P P}^{k} \leq \lambda_{B P}^{k}$ and $w_{k} \lambda_{P P}^{k} \leq w_{k} \lambda_{B P}^{k}$. The relationship $m_{P P} \leq m_{B P}$ is true. Analogously, we can deduce the following relationships: $m_{P P} \leq m_{B P}<m_{N P}$ and $m_{N N} \leq m_{B N}<m_{P N}$. Hence, the statement of Proposition 1 holds.

For (4) and (5), there are two conflicting requirements: (i) One is the justifiable granularity, i.e., $\sum_{l_{\Delta \nabla} \leq y \leq m_{\Delta \nabla}} w_{\Delta \nabla}^{\sigma(N(y))}$ and $\sum_{m_{\Delta \nabla} \leq y \leq u_{\Delta \nabla}} w_{\Delta \nabla}^{\sigma(N(y))}$ satisfy the requirement that the number of data falling within $\lambda_{\Delta \nabla}$ is as high as possible; (ii) The other is the specificity, i.e., $\exp \left(-\varepsilon\left|m_{\Delta \nabla}-l_{\Delta \nabla}\right|\right)$ and $\exp \left(-\varepsilon\left|m_{\Delta \nabla}-u_{\Delta \nabla}\right|\right)$ satisfy the requirement that the support of $\lambda_{\Delta \nabla}$ is as low as possible. To obtain the important and majority suggestions of experts and improve the inconsistency of GDM, we aggregate the group decision result of $\lambda_{\Delta \nabla}$ with an interval. When the value of $\varepsilon$ is constant, we determine the interval $\lambda_{\Delta \nabla}=\left[\lambda_{\Delta \nabla}^{-}, \lambda_{\Delta \nabla}^{+}\right]$by optimizing (4) and (5), i.e.,

$$
\lambda_{\Delta \nabla}^{-}=\underset{l_{\Delta \nabla} \leq m_{\Delta \nabla}}{\arg \max } V\left(l_{\Delta \nabla}\right)
$$




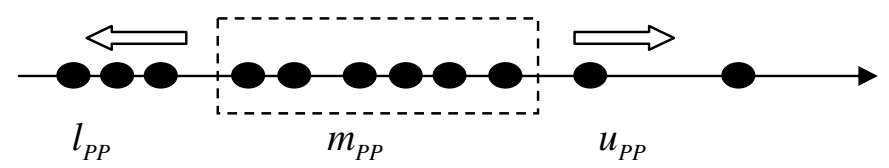

Figure 2: The information granulation sketch of loss function $\lambda_{P P}$

$$
\lambda_{\Delta \nabla}^{+}=\underset{m_{\Delta \nabla} \leq u_{\Delta \nabla}}{\arg \max } V\left(u_{\Delta \nabla}\right)
$$

From (4)-(8), the information granulation of loss function depends on the distribution of the evaluations and the weights of the experts. To make it clear, we simply visualize the information granulation sketch of loss function $\lambda_{P P}$ as an example. Its sketch is shown in Fig. 2.

In Fig.2, the black dots presented in the x-coordinate denote the evaluation results of experts. Two arrows above the $\mathrm{x}$-coordinate describe the optimization directions based on the mean, which are used to search the lower bound and the upper bound of interval. The range with a dotted line denotes an interval information granule. Toward the left, it is used to determine the lower bound. The other direction is used to calculate the upper bound. If the composite multiplicative indexes (6)-(7) of the lower bound and the upper bound are zero, we directly use the mean to replace them. With the aid of the principle of justifiable granularity, we finally gain the interval information granule of each loss function below: $\lambda_{P P}=\left[\lambda_{P P}^{-}, \lambda_{P P}^{+}\right], \lambda_{B P}=\left[\lambda_{B P}^{-}, \lambda_{B P}^{+}\right], \lambda_{N P}=\left[\lambda_{N P}^{-}, \lambda_{N P}^{+}\right], \lambda_{P N}=\left[\lambda_{P N}^{-}, \lambda_{P N}^{+}\right], \lambda_{B N}=\left[\lambda_{B N}^{-}, \lambda_{B N}^{+}\right]$, $\lambda_{N N}=\left[\lambda_{N N}^{-}, \lambda_{N N}^{+}\right]$. Obviously, we further deduce the relationship between the lower bound (upper bound) of loss function and its weighted mean.

Proposition 2. Based on (6)-(8), the relationship between the lower bound (upper bound) of loss function and its weighted mean is: $\lambda_{\Delta \nabla}^{-} \leq m_{\Delta \nabla} \leq \lambda_{\Delta \nabla}^{+}(\triangle=P, B, N ; \nabla=P, N)$.

On the basis of the information granulation of loss function, we use an example to elaborate it as follows.

Example 1. In order to illustrate the calculation procedure of the information granulation of loss function, we take the loss function $\lambda_{P N}$ as an example. The evaluation results of $\lambda_{P N}$ under the GDM situation have: $\lambda_{P N}^{1}=40 u, \lambda_{P N}^{2}=14 u$, $\lambda_{P N}^{3}=20 u, \lambda_{P N}^{4}=30 u, \lambda_{P N}^{5}=18 u, \lambda_{P N}^{6}=20 u, \lambda_{P N}^{7}=23 u, \lambda_{P N}^{8}=21 u, \lambda_{P N}^{9}=24 u, \lambda_{P N}^{10}=20 u, \lambda_{P N}^{11}=24 u . T h e$ corresponding weight vector of experts is given as follows: $W=\left\{w_{1}, w_{2}, w_{3}, w_{4}, w_{5}, w_{6}, w_{7}, w_{8}, w_{9}, w_{10}, w_{11}\right\}^{T}=$ $\{0.05,0.05,0.05,0.05,0.05,0.05,0.1,0.1,0.1,0.2,0.2\}^{T}$. For the evaluation results of $\lambda_{P N}$, we merge the same values and sort them in an ascending order. The results are:

$$
\lambda_{P N}^{\sigma(1)}=14 u, \lambda_{P N}^{\sigma(2)}=18 u, \quad \lambda_{P N}^{\sigma(3)}=20 u, \quad \lambda_{P N}^{\sigma(4)}=21 u, \quad \lambda_{P N}^{\sigma(5)}=23 u, \quad \lambda_{P N}^{\sigma(6)}=24 u, \quad \lambda_{P N}^{\sigma(7)}=30 u, \quad \lambda_{P N}^{\sigma(8)}=40 u .
$$

At present, the corresponding weight information is:

$$
w_{P N}^{\sigma(1)}=0.05, w_{P N}^{\sigma(2)}=0.05, w_{P N}^{\sigma(3)}=0.3, w_{P N}^{\sigma(4)}=0.1, w_{P N}^{\sigma(5)}=0.1, \quad w_{P N}^{\sigma(6)}=0.3, \quad w_{P N}^{\sigma(7)}=0.05, \quad w_{P N}^{\sigma(8)}=0.05
$$

Under a certain value of $\varepsilon$, we utilize (4)-(8) to calculate the composite multiplicative index for each evaluation outcome. Considering the value of $\varepsilon$ is 0.03 , the mean is $m_{P N}=22.7 u$. As the sketch presented in Fig. 2, we calculate the composite multiplicative index toward the left of $m_{P N}$, i.e., $V\left(\lambda_{P N}^{\sigma(1)}\right)=0.3851, V\left(\lambda_{P N}^{\sigma(2)}\right)=0.3908, V\left(\lambda_{P N}^{\sigma(3)}\right)=0.3689$ and $V\left(\lambda_{P N}^{\sigma(4)}\right)=0.0950$. When the evaluation result is $18 u$, we find its composite multiplicative index in the left of $m_{P N}$ is maximum and determine the lower bound of interval. Similarly, when the evaluation result is $24 u$, we find that its composite multiplicative index in the right of $m_{P N}$ is maximum. At this point, we generate an interval-valued information granule of $\lambda_{P N}$, namely, $[18 u, 24 u]$.

On basis of the results shown in [37], the essential role of $\varepsilon$ is to calibrate an impact of the specificity criterion on the constructed information granule. With the variation of $\varepsilon$, the change of the information granule of loss function is discussed in the following proposition.

Proposition 3. When the evaluation results of loss function $\lambda_{\Delta \nabla}$ and the weight of experts $W=\left\{w_{1}, w_{2}, \cdots, w_{t}\right\}^{T}$ are constant, the distance between the lower and upper bounds of the information granule of loss function, i.e., $\left|\lambda_{\Delta \nabla}^{+}-\lambda_{\Delta \nabla}^{-}\right|$, can be amplified or invariant with the decreasing of $\varepsilon(\triangle=P, B, N ; \nabla=P, N)$. 


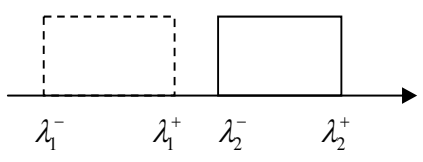

(a)

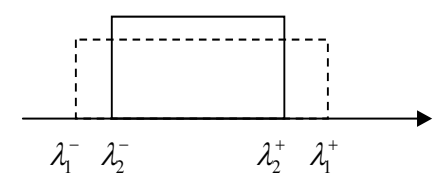

(c)

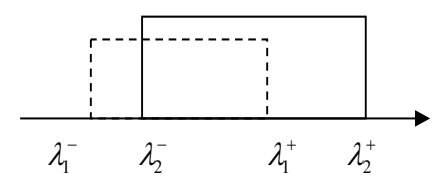

(e)

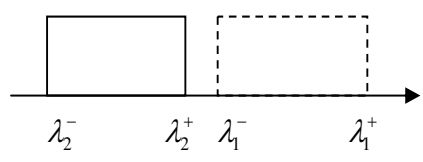

(b)

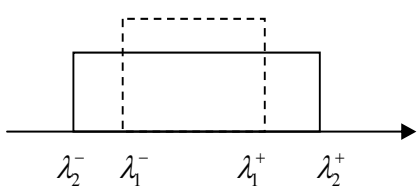

(d)

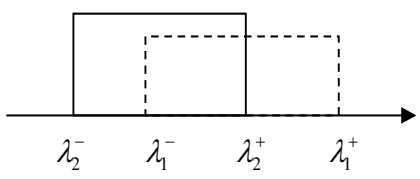

(f)

Figure 3: Some possible relationships between $\lambda_{1}=\left[\lambda_{1}^{-}, \lambda_{1}^{+}\right]$and $\lambda_{2}=\left[\lambda_{2}^{-}, \lambda_{2}^{+}\right]$

Table 5: The possible relationships between the interval information granules of loss functions

\begin{tabular}{|c|c|c|c|c|c|c|c|}
\hline The loss functions on two states & Comparison between two loss functions & (a) & (b) & (c) & (d) & (e) & (f) \\
\hline \multirow{2}{*}{$\lambda_{P P}, \lambda_{B P}, \lambda_{N P}$} & $\lambda_{P P}$ and $\lambda_{B P}$ & $\sqrt{ }$ & & $\sqrt{ }$ & $\sqrt{ }$ & $\sqrt{ }$ & $\sqrt{ }$ \\
& $\lambda_{B P}$ and $\lambda_{N P}$ & $\sqrt{ }$ & & $\sqrt{ }$ & $\sqrt{ }$ & $\sqrt{ }$ & $\sqrt{ }$ \\
\hline \multirow{2}{*}{$\lambda_{P N}, \lambda_{B N}, \lambda_{N N}$} & $\lambda_{P N}$ and $\lambda_{B N}$ & $\sqrt{ }$ & & $\sqrt{ }$ & $\sqrt{ }$ & $\sqrt{ }$ & $\sqrt{ }$ \\
& $\lambda_{B N}$ and $\lambda_{N N}$ & $\sqrt{ }$ & & $\sqrt{ }$ & $\sqrt{ }$ & $\sqrt{ }$ & $\sqrt{ }$ \\
\hline
\end{tabular}

Note: In the table, $\sqrt{ }$ denotes the applicability.

Proof. With respect to the loss function $\lambda_{\Delta \nabla}, \exp \left(-\varepsilon\left|m_{\Delta \nabla}-l_{\Delta \nabla}\right|\right)$ and $\exp \left(-\varepsilon\left|m_{\Delta \nabla}-u_{\Delta \nabla}\right|\right)$ of (4)-(5) are increasing with decreasing of $\varepsilon$. Given the value of $y, \sum_{l_{\Delta \nabla} \leq y \leq m_{\Delta \nabla}} w_{\Delta \nabla}^{\sigma(N(y))}$ and $\sum_{m_{\Delta \nabla} \leq y \leq u_{\Delta \nabla}} w_{\Delta \nabla}^{\sigma(N(y))}$ are constant. In this case, $\exp \left(-\varepsilon\left|m_{\Delta \nabla}-l_{\Delta \nabla}\right|\right)$ and $\exp \left(-\varepsilon\left|m_{\Delta \nabla}-u_{\Delta \nabla}\right|\right)$ become main factors in the determination of $V\left(l_{\Delta \nabla}\right)$ and $V\left(u_{\Delta \nabla}\right)$. When $l_{\Delta \nabla} \leq y \leq m_{\Delta \nabla}, V\left(l_{\Delta \nabla}\right) \geq V(y)$. Considering the decreasing of $\varepsilon$, the relationship between $V\left(l_{\Delta \nabla}\right)$ and $V(y)$ depends on $\exp \left(-\varepsilon\left|m_{\Delta \nabla}-l_{\Delta \nabla}\right|\right)$ and $\sum_{l_{\Delta \nabla} \leq y \leq m_{\Delta \nabla}} w_{\Delta \nabla}^{\sigma(N(y))}$. If $y \leq l_{\Delta \nabla}, V\left(l_{\Delta \nabla}\right) \geq V(y)$ or $V\left(l_{\Delta \nabla}\right) \leq V(y)$. According to the above discussions, the lower bound may be becoming more and more smaller. It gives rise to the expansion of the distance between the lower bound of information granule and the weighted mean or keep the constant. Similarly, the distance between the upper bound of information granule and the weighted mean can be amplified or keep the constant. Thus, the statement in Proposition 3 holds.

Proposition 3 also implies that the distance between the lower and upper bounds of the information granule of loss function may be shrunk with the increasing of $\varepsilon$. Given two intervals $\lambda_{1}=\left[\lambda_{1}^{-}, \lambda_{1}^{+}\right]$and $\lambda_{2}=\left[\lambda_{2}^{-}, \lambda_{2}^{+}\right]$, their possible relationships are depicted in Fig. 3.

In Fig. 3 , the dotted line and the solid line denote the intervals $\lambda_{1}=\left[\lambda_{1}^{-}, \lambda_{1}^{+}\right]$and $\lambda_{2}=\left[\lambda_{2}^{-}, \lambda_{2}^{+}\right]$, respectively. The possible relationships of the two intervals are listed, i.e., (a)-(f). For the information granules of loss functions, they are intervals. In light of (4)-(8), the information granulation process mainly relies on the mean and the distribution of the evaluations in the GDM. The possible relationships between the interval information granules of loss functions are summarized in Table 5.

According to the result presented in Proposition 1, (b) of Fig. 3 is not suitable to the loss functions. When $\varepsilon$ 
Table 6: The interval-valued loss functions regarding the risk or cost of actions in different states

\begin{tabular}{|c|c|c|}
\hline & $C(P)$ & $\neg C(N)$ \\
\hline$a_{P}$ & $\lambda_{P P}=\left[\lambda_{P P}^{-}, \lambda_{P P}^{+}\right]$ & $\lambda_{P N}=\left[\lambda_{P N}^{-}, \lambda_{P N}^{+}\right]$ \\
$a_{B}$ & $\lambda_{B P}=\left[\lambda_{B P}^{-}, \lambda_{B P}^{+}\right]$ & $\lambda_{B N}=\left[\lambda_{B N}^{-}, \lambda_{B N}^{+}\right]$ \\
$a_{N}$ & $\lambda_{N P}=\left[\lambda_{N P}^{-}, \lambda_{N P}^{+}\right]$ & $\lambda_{N N}=\left[\lambda_{N N}^{-}, \lambda_{N N}^{+}\right]$ \\
\hline
\end{tabular}

is constant, other cases of Fig. 3 may be existed. Based on (1) and (2), Liang et al. [21] discussed the semantics and stipulated the relationships among the interval-valued loss functions, i.e., $\lambda_{P P}^{-} \leq \lambda_{B P}^{-}<\lambda_{N P}^{-}, \lambda_{P P}^{+} \leq \lambda_{B P}^{+}<\lambda_{N P}^{+}$, $\lambda_{N N}^{-} \leq \lambda_{B N}^{-}<\lambda_{P N}^{-}$and $\lambda_{N N}^{+} \leq \lambda_{B N}^{+}<\lambda_{P N}^{+}$. Obviously, some cases of Fig. 3 are inconsistent with the results reported in [21], e.g., (d) and (f). Inspired by Proposition 3, the inconsistency can be improved by rectifying the value of $\varepsilon$.

Proposition 4. Suppose the evaluation results of loss function $\lambda_{\Delta \nabla}$ and the weight of experts $W=\left\{w_{1}, w_{2}, \cdots, w_{t}\right\}^{T}$ are constant. If $\varepsilon \rightarrow 0$, the relationships among the information granules of loss functions $\lambda_{\Delta \nabla}=\left[\lambda_{\Delta \nabla}^{-}, \lambda_{\Delta \nabla}^{+}\right]$based on (1) and (2) have $(\triangle=P, B, N ; \nabla=P, N)$ :

$$
\begin{aligned}
\lambda_{P P}^{-} & \leq \lambda_{B P}^{-}<\lambda_{N P}^{-}, \\
\lambda_{P P}^{+} & \leq \lambda_{B P}^{+}<\lambda_{N P}^{+}, \\
\lambda_{N N}^{-} & \leq \lambda_{B N}^{-}<\lambda_{P N}^{-}, \\
\lambda_{N N}^{+} & \leq \lambda_{B N}^{+}<\lambda_{P N}^{+} .
\end{aligned}
$$

According to the results presented in Propositions 3 and 4, we can obtain some suitable loss functions to keep the consistent relationship with [21] by adjusting the value of $\varepsilon$. This is regarded as an interactive process. Finally, the interval-valued information granules of the loss functions regarding the risk or cost of actions in the different states are given by a $3 \times 2$ matrix in Table 6 .

\subsection{Three-way decisions derived from interval-valued loss functions}

According to the results presented in Yao [50], three-way decisions with DTRSs strictly comply with the Bayesian decision procedure. Through the comparison of the expected losses, three-way decisions generate judgment criteria and determine the action for each object, see the decision rules $(\mathrm{P})-(\mathrm{N})$. For the loss function matrix presented in Table 6, each loss function is an interval. For the interval analysis, Liang et al. [21] summarized some typical analysis methods and compared them in the context of three-way decisions. According to the results reported in [21], the $\theta$ ranking method proposed by Liu et al. [26] is suitable to compare the interval-valued loss functions. In the following discussions, we choose the $\theta$ ranking method to deduce three-way decisions. The method can transform the intervals into real numbers and is also easy to implement [22]. The transformed formula is described as follows [26].

Definition 1. Let an interval $\lambda=\left[\lambda^{-}, \lambda^{+}\right], \theta \in[0,1]$, the transformed formula of $\lambda$ is:

$$
m_{\theta}(\lambda)=(1-\theta) \lambda^{-}+\theta \lambda^{+} .
$$

where $m_{\theta}(\lambda)$ is the transformed outcome and $\theta$ reflects the risk attitude of decision makers.

From (11), $m_{\theta}(\lambda)$ is a real number. In light of the results presented in [21], a larger value of $\theta$ indicates a lower degree of optimism. More specifically, when the conditions $\theta=0$ and $\theta=1$ hold, the value of $m_{\theta}(\lambda)$ represents an optimistic and a pessimistic viewpoints. When $\theta$ is 0.5 , the value of $m_{\theta}(\lambda)$ denotes a viewpoint of a moderate decision maker. With the aid of the $\theta$ ranking method, the comparison principle between intervals is shown as follows [26].

Definition 2. Given two intervals $\lambda_{1}=\left[\lambda_{1}^{-}, \lambda_{1}^{+}\right], \lambda_{2}=\left[\lambda_{2}^{-}, \lambda_{2}^{+}\right]$. Suppose the value of $\theta$ is a constant. If $m_{\theta}\left(\lambda_{1}\right) \leq$ $m_{\theta}\left(\lambda_{2}\right), \lambda_{1} \leq \lambda_{2}$, and vice versa. 
On basis of Definitions 1 and 2, we confirm the values of loss functions presented in Table 6, i.e.,

$$
m_{\theta}\left(\lambda_{\Delta \nabla}\right)=(1-\theta) \lambda_{\Delta \nabla}^{-}+\theta \lambda_{\Delta \nabla}^{+}
$$

In this situation, the results of loss functions are shown: $m_{\theta}\left(\lambda_{P P}\right), m_{\theta}\left(\lambda_{B P}\right), m_{\theta}\left(\lambda_{N P}\right), m_{\theta}\left(\lambda_{P N}\right), m_{\theta}\left(\lambda_{B N}\right)$ and $m_{\theta}\left(\lambda_{N N}\right)$. With respect to (3), the thresholds values are recalculated as:

$$
\begin{aligned}
\alpha & =\frac{\left(m_{\theta}\left(\lambda_{P N}\right)-m_{\theta}\left(\lambda_{B N}\right)\right)}{\left(m_{\theta}\left(\lambda_{P N}\right)-m_{\theta}\left(\lambda_{B N}\right)\right)+\left(m_{\theta}\left(\lambda_{B P}\right)-m_{\theta}\left(\lambda_{P P}\right)\right)}, \\
\beta & =\frac{\left(m_{\theta}\left(\lambda_{B N}\right)-m_{\theta}\left(\lambda_{N N}\right)\right.}{\left(m_{\theta}\left(\lambda_{B N}\right)-m_{\theta}\left(\lambda_{N N}\right)\right)+\left(m_{\theta}\left(\lambda_{N P}\right)-m_{\theta}\left(\lambda_{B P}\right)\right)}, \\
\gamma & =\frac{\left(m_{\theta}\left(\lambda_{P N}\right)-m_{\theta}\left(\lambda_{N N}\right)\right)}{\left(m_{\theta}\left(\lambda_{P N}\right)-m_{\theta}\left(\lambda_{N N}\right)\right)+\left(m_{\theta}\left(\lambda_{N P}\right)-m_{\theta}\left(\lambda_{P P}\right)\right)} .
\end{aligned}
$$

According to the discussions presented in $[21,28,50,51]$, we further simplify decision rules $(\mathrm{P})$-(N). Considering the conditions of decision rule (B), they suggest that $\alpha>\beta$, that is,

$$
\frac{\left(m_{\theta}\left(\lambda_{B P}\right)-m_{\theta}\left(\lambda_{P P}\right)\right)}{\left(m_{\theta}\left(\lambda_{P N}\right)-m_{\theta}\left(\lambda_{B N}\right)\right)}<\frac{\left(m_{\theta}\left(\lambda_{N P}\right)-m_{\theta}\left(\lambda_{B P}\right)\right)}{\left(m_{\theta}\left(\lambda_{B N}\right)-m_{\theta}\left(\lambda_{N N}\right)\right)} .
$$

It implies $0 \leq \beta<\gamma<\alpha \leq 1$. In this case, after tie-breaking, the following simplified rules are obtained:

$$
\text { If } \operatorname{Pr}(C \mid[x]) \geq \alpha \text {, decide } x \in \operatorname{POS}(C) \text {; }
$$$$
\text { If } \beta<\operatorname{Pr}(C \mid[x])<\alpha \text {, decide } x \in \operatorname{BND}(C) \text {; }
$$$$
\text { If } \operatorname{Pr}(C \mid[x]) \leq \beta \text {, decide } x \in \operatorname{NEG}(C) \text {. }
$$

In addition, we also can consider another condition $\alpha \leq \beta$, that is,

$$
\frac{\left(m_{\theta}\left(\lambda_{B P}\right)-m_{\theta}\left(\lambda_{P P}\right)\right)}{\left(m_{\theta}\left(\lambda_{P N}\right)-m_{\theta}\left(\lambda_{B N}\right)\right)} \geq \frac{\left(m_{\theta}\left(\lambda_{N P}\right)-m_{\theta}\left(\lambda_{B P}\right)\right)}{\left(m_{\theta}\left(\lambda_{B N}\right)-m_{\theta}\left(\lambda_{N N}\right)\right)} .
$$

It implies $0 \leq \alpha \leq \gamma \leq \beta \leq 1$. In this case, after tie-breaking, the following simplified rules are obtained:

$$
\text { If } \operatorname{Pr}(C \mid[x]) \geq \gamma \text {, decide } x \in \operatorname{POS}(C) \text {; }
$$

$$
\text { If } \operatorname{Pr}(C \mid[x])<\gamma \text {, decide } x \in \operatorname{NEG}(C) \text {. }
$$

In light of decision rules (P1)-(N1) and (P2)-(N2), one can discriminate the decision action of each object $x$.

\subsection{The decision procedure of GDM-based three-way decisions}

With the help of the above-mentioned results, we design GDM-based three-way decisions in this subsection. The key steps are elaborated as follows.

Step 1: Under the GDM context, determine the number of experts and their own weight, in which the set of experts is denoted as $E=\left\{e_{1}, e_{2}, \cdots, e_{t}\right\}$ and the weight vector of experts is $W=\left\{w_{1}, w_{2}, \cdots, w_{t}\right\}^{T}$.

Step 2: According to the loss functions presented in Table 2, each expert provides his/her evaluation. The gathering evaluation information can be denoted as $\lambda_{P P}^{k}, \lambda_{B P}^{k}, \lambda_{N P}^{k}, \lambda_{P N}^{k}, \lambda_{B N}^{k}$ and $\lambda_{N N}^{k}(1 \leq k \leq t)$.

Step 3: For aggregating the experts' suggestions and improving the inconsistency among the experts, given the value of $\varepsilon$, we generate the interval-valued information granule for each loss function based on (4)-(8), i.e., $\lambda_{P P}=$ $\left[\lambda_{P P}^{-}, \lambda_{P P}^{+}\right], \lambda_{B P}=\left[\lambda_{B P}^{-}, \lambda_{B P}^{+}\right], \lambda_{N P}=\left[\lambda_{N P}^{-}, \lambda_{N P}^{+}\right], \lambda_{P N}=\left[\lambda_{P N}^{-}, \lambda_{P N}^{+}\right], \lambda_{B N}=\left[\lambda_{B N}^{-}, \lambda_{B N}^{+}\right], \lambda_{N N}=\left[\lambda_{N N}^{-}, \lambda_{N N}^{+}\right]$.

Step 4: If the interval-valued loss functions satisfy the conditions of Proposition 4 , determine the loss functions and go to Step 5. Otherwise, we need to assign a new value for $\varepsilon$ and go to Step 3.

Step 5: By using (9), transform the interval-valued information granules with a certain value of $\theta$ and determine the precise values of loss functions. The results are denoted as: $m_{\theta}\left(\lambda_{P P}\right), m_{\theta}\left(\lambda_{B P}\right), m_{\theta}\left(\lambda_{N P}\right), m_{\theta}\left(\lambda_{P N}\right), m_{\theta}\left(\lambda_{B N}\right)$ and $m_{\theta}\left(\lambda_{N N}\right)$.

Step 6: Based on (10), calculate the thresholds $(\alpha, \beta, \gamma)$ 


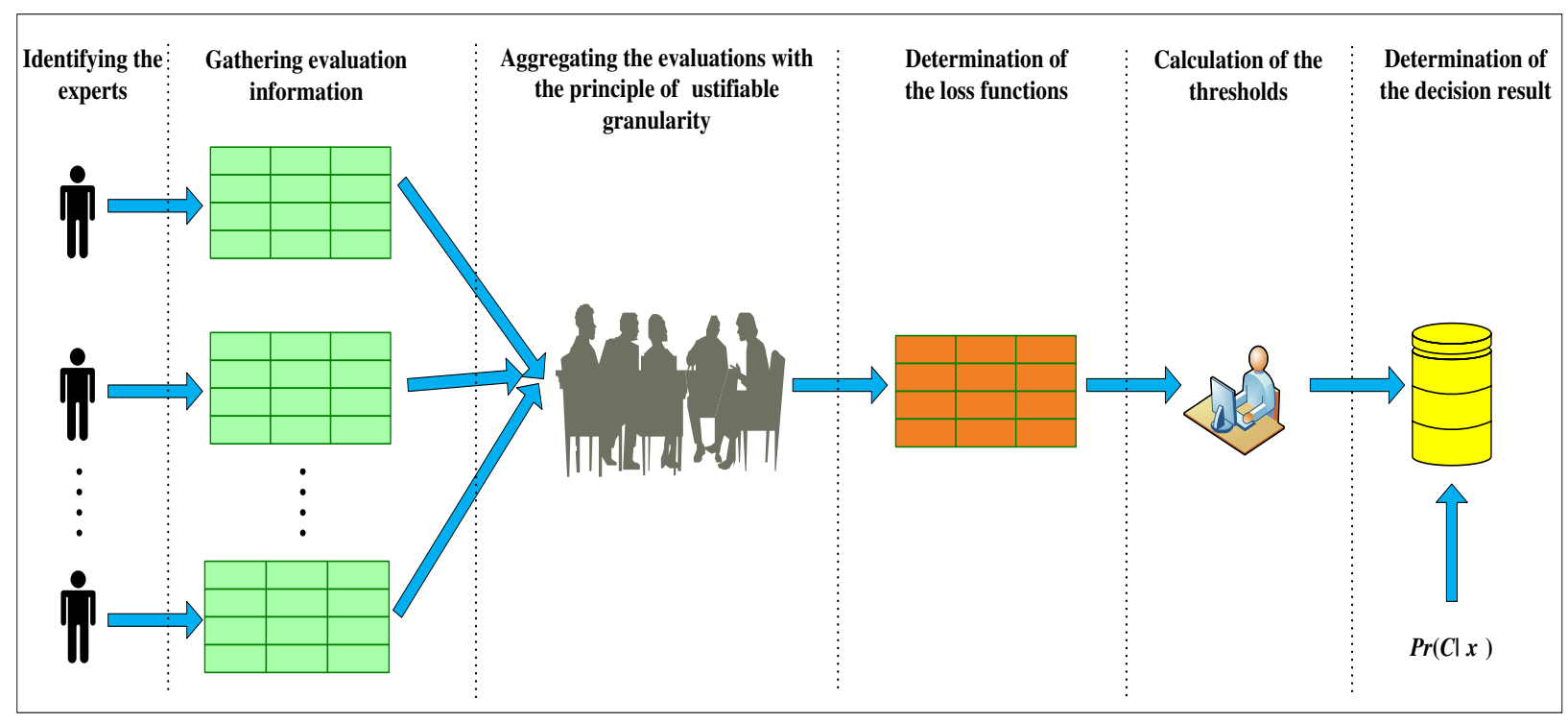

Figure 4: The scheme of GDM-based three-way decisions

Step 7: Through the comparison of (11) and (12), obtain the relationships among loss functions and confirm the corresponding judgement criteria ((P1)-(N1) or (P2)-(N2)). Given the conditional probability, i.e., $\operatorname{Pr}(C \mid[x])$, we can discriminate the decision action of each object.

For clarity, the scheme of GDM-based three-way decisions is shown in Fig. 4. Note that the relationships among the information granules of loss functions must satisfy the conditions of Proposition 4. If not, we need to reconsider the value of $\varepsilon$ and implement Step 3. In light of the results presented in [20], our proposed method mainly employs the novel idea of granular computing. First, the information granulation has been used in the determination of loss functions. When we deduce the three-way decisions, we further adopt the information degranulation replied on (9).

\section{An illustration example}

In this section, we utilize an example of strategic supplier selection to illustrate GDM-based three-way decisions. The supply management is one of the key issues of a manufacturing firm, which directly impacts the production on time. For example, when the supply process of raw materials delays, it may affect the sales revenue of the firm. Suppliers have been acknowledged as the best intangible assets of any business organization. Selecting the right suppliers for a long term relationship is a relevant procurement issue that demands judicious attention. Thus, the strategic supplier selection for a firm is crucial, which impacts an effective supply management. During the evaluation, the strategic supplier selection is impacted by numerous risk and uncertain factors $[1,3,5,6,9,10,11,13]$. For example, Büyüközkan et al. [3] evaluated the strategic alliance partner from the strategic dimension and the business excellence dimension. Ho et al. [13] listed evaluating criteria, i.e., cost, delivery, flexibility, quality, technology and risk. They also summarized most important evaluating criteria and some evaluation approaches. Under the risk environment, the selection of appropriate suppliers may consider the losses existing in some possible decision results. In order to correctly evaluate strategic suppliers, there is a need to invite multiple experts, which is regarded as a GDM problem [13]. GDM-based three-way decisions exactly satisfy the requirements of strategic supplier selection. Except for the selection and non-selection strategies, the deferred action of the strategic supplier is added into the strategy. More importantly, the method integrates risk factors and also considers that some possible decision results of the selection may result in different losses. The decision procedure of GDM-based three-way decisions for the strategic supplier selection is designed in Fig. 5.

In Fig. 5, there are two parts: problem formulation and decision analysis. According to the context of strategic supplier selection, we firstly define the concepts of the relevant parameters of GDM-based three-way decisions. 


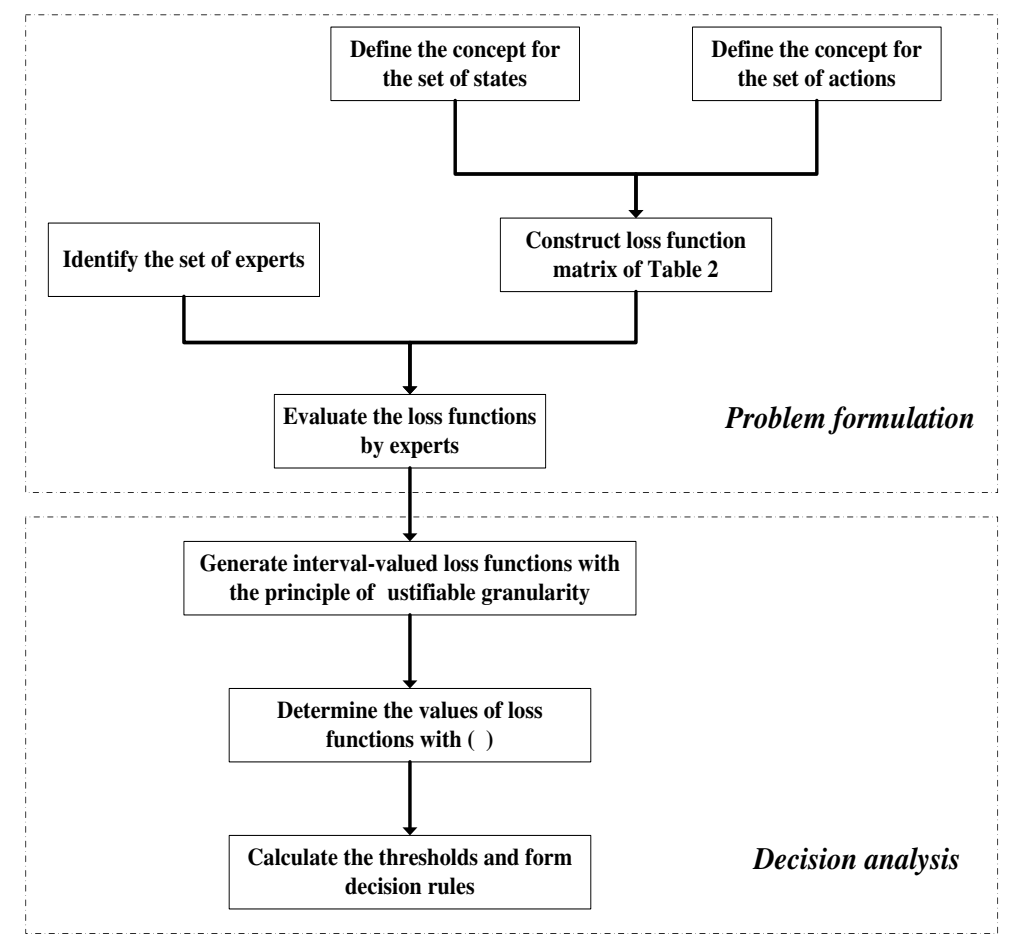

Figure 5: The decision procedure of GDM-based three-way decisions for the strategic supplier selection

Meanwhile, we also need to determine the expert information and collect their evaluations. Based on the evaluation results, we are engaged in decision analysis. For the strategic supplier selection problem, we have two states $\Omega=\{C, \neg C\}$ indicating that the supplier is good or bad, respectively. The set of actions for the supplier $x$ is given by $\mathcal{A}=\left\{a_{P}, a_{B}, a_{N}\right\}$, where $a_{P}, a_{B}$, and $a_{N}$ represent the selection, the deferment (non-commitment) decision and the rejection decision. The conditional probability $\operatorname{Pr}(C \mid[x])$ is the probability of a supplier $x$ being a good supplier, which is computed by the past similar ideas described by its equivalence class $[x]$. The existing literatures of the strategic supplier selection mainly discuss the evaluation of the conditional probability $[1,3,5,6,9,10,11,13]$. In this example, we assume that $\operatorname{Pr}(C \mid[x])$ is known and focus on discussing the loss functions. In the three-way decisions, we consider six types of loss functions, i.e., $\lambda_{P P}, \lambda_{B P}, \lambda_{N P}, \lambda_{P N}, \lambda_{B N}$ and $\lambda_{N N}$. On basis of the results presented in Table 2, $\lambda_{P P}, \lambda_{B P}$ and $\lambda_{N P}$ denote the losses incurred for taking actions of the selection, the deferment (non-commitment) decision and the rejection decision respectively, when the supplier belongs to a good supplier. Similarly, $\lambda_{P N}, \lambda_{B N}$ and $\lambda_{N N}$ denote the losses incurred for taking actions of the selection, the deferment (non-commitment) decision and the rejection decision when the supplier is a bad one.

Step 1: For making reasonable selection decisions, relevant experts are invited to evaluate the loss functions. The set of experts is $E=\left\{e_{1}, e_{2}, e_{3}, e_{4}, e_{5}, e_{6}, e_{7}, e_{8}, e_{9}, e_{10}, e_{11}\right\}$ and the corresponding weight vector of experts is $W=\left\{w_{1}, w_{2}, w_{3}, w_{4}, w_{5}, w_{6}, w_{7}, w_{8}, w_{9}, w_{10}, w_{11}\right\}^{T}=\{0.05,0.05,0.05,0.05,0.05,0.05,0.1,0.1,0.1,0.2,0.2\}^{T}$.

Step 2: In light of the results reported in Table 2, each expert assigns his (or her) own estimated values of loss functions. The evaluation results of experts are summarized in Table 7.

Step 3: Following GDM-based three-way decisions, we granulate the loss function $\lambda_{\Delta \nabla}$ and determine its lower and upper bound $(\triangle=P, B, N ; \nabla=P, N)$.

From Table 7, the weighted means of loss functions are calculated as: $m_{P P}=0.88 u, m_{B P}=13.25 u, m_{N P}=26.75 u$, $m_{P N}=22.7 u, m_{B N}=11.91 u$ and $m_{N N}=1.635 u$. Considering the different opinions among experts, we express the group decision result with an interval-valued format by using (4)-(8). When the value of $\varepsilon$ is certain, we can get the interval information granules of loss functions with the aid of the principle of justifiable granularity. Given that the value of $\varepsilon$ is 1, the process of information granulation of each loss function is described in Fig. 6. 
Table 7: The evaluation results under the group decision making context ( $u$ is a unit cost)

\begin{tabular}{|c|c|c|c|c|c|c|}
\hline$E$ & $\lambda_{P P}$ & $\lambda_{B P}$ & $\lambda_{N P}$ & $\lambda_{P N}$ & $\lambda_{B N}$ & $\lambda_{N N}$ \\
\hline$e_{1}$ & $1.5 u$ & $4 u$ & $8 u$ & $40 u$ & $8.5 u$ & 0 \\
$e_{2}$ & $2 u$ & $9 u$ & $14 u$ & $14 u$ & $9 u$ & 0 \\
$e_{3}$ & $u$ & $10.1 u$ & $15 u$ & $20 u$ & $9.2 u$ & $0.5 u$ \\
$e_{4}$ & $u$ & $10 u$ & $16 u$ & $30 u$ & $9.5 u$ & $0.8 u$ \\
$e_{5}$ & $0.05 u$ & $10.2 u$ & $14 u$ & $18 u$ & $10 u$ & $u$ \\
$e_{6}$ & $1.05 u$ & $10.2 u$ & $20 u$ & $20 u$ & $10 u$ & $u$ \\
$e_{7}$ & $2 u$ & $14.2 u$ & $23 u$ & $21 u$ & $10 u$ & $1.2 u$ \\
$e_{8}$ & $2.5 u$ & $11 u$ & $21 u$ & $23 u$ & $10 u$ & $1.5 u$ \\
$e_{9}$ & $u$ & $12 u$ & $28 u$ & $24 u$ & $11 u$ & $2 u$ \\
$e_{10}$ & 0 & $20 u$ & $34 u$ & $20 u$ & $12 u$ & $2 u$ \\
$e_{11}$ & 0 & $15 u$ & $41 u$ & $24 u$ & $18 u$ & $3 u$ \\
\hline
\end{tabular}
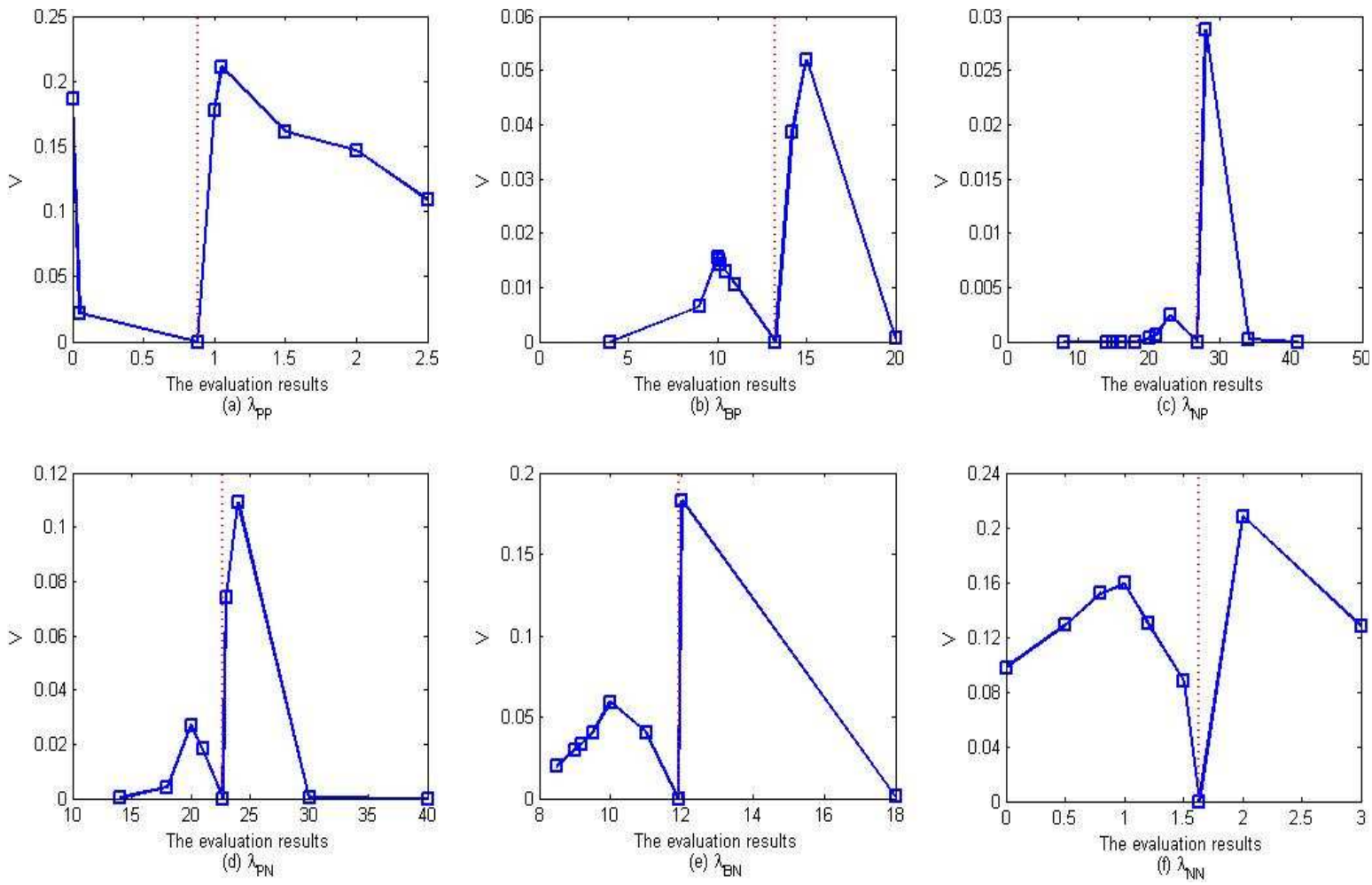

Figure 6: The process of information granulation of each loss function with $\varepsilon=1$ ( $u$ is a unit cost) 


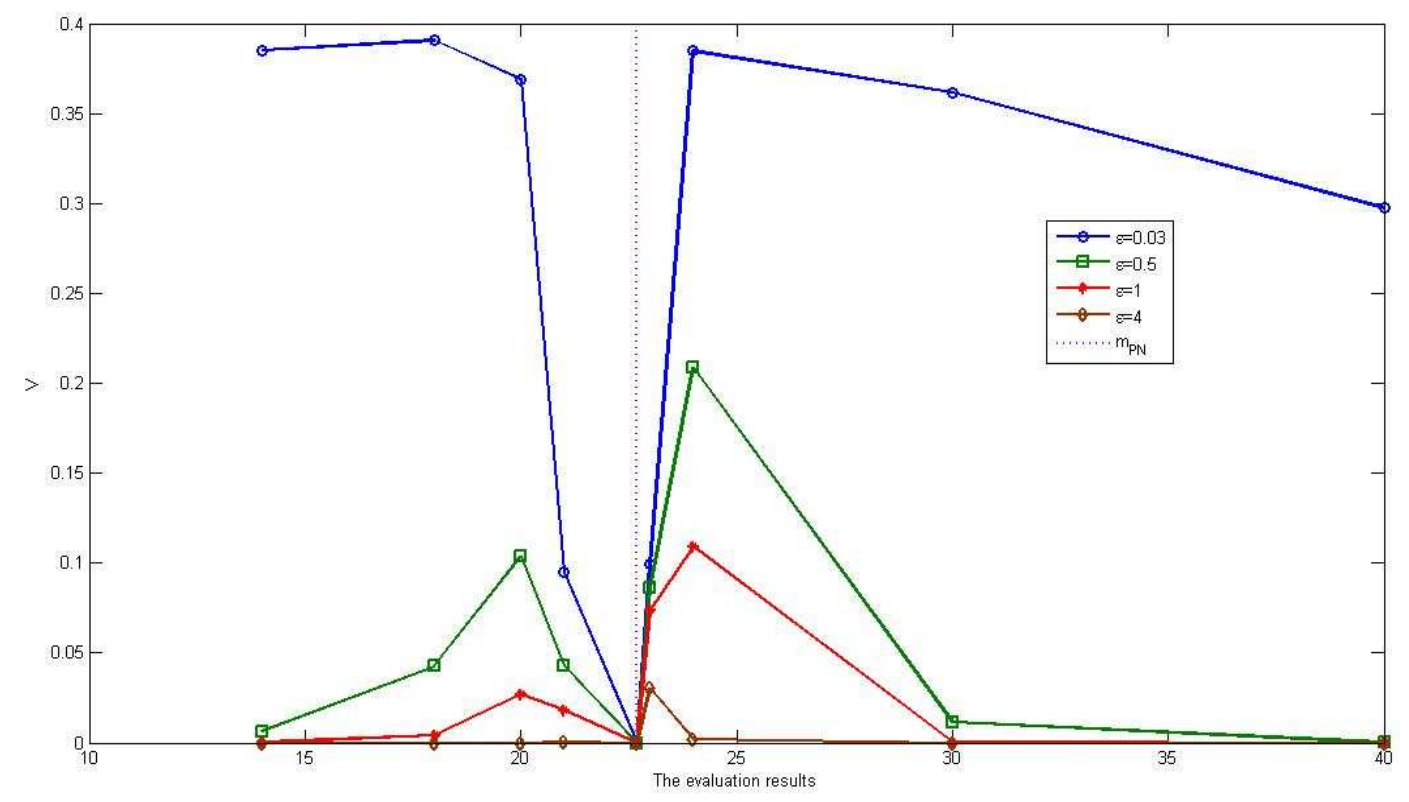

Figure 7: The granulation procedure of the evaluation results of $\lambda_{P N}$ ( $u$ is a unit cost)

In Fig. 6, the dotted lines stand for the weighted means of loss functions. Based on the weighted means and (4)-(8), we search the lower and the upper bounds for each loss function. The solid lines denote the composite multiplicative indexes of each evaluation result of experts, which describes the granulation procedure of the evaluation results. In light of Example 1, we take the loss function $\lambda_{P N}$ as an example to state the procedure. According to the results presented in Table 7, the evaluation results of $\lambda_{P N}$ under the GDM situation have: $\lambda_{P N}^{1}=40 u, \lambda_{P N}^{2}=14 u, \lambda_{P N}^{3}=20 u$, $\lambda_{P N}^{4}=30 u, \cdots, \lambda_{P N}^{11}=24 u$. Meanwhile, we obtain the corresponding weight information: $w_{1}=0.05, w_{2}=0.05$, $w_{3}=0.05, w_{4}=0.05, \cdots, w_{11}=0.2$. For the evaluation results of $\lambda_{P N}$, we merge the same values and sort them in an ascending order. Continued the analysis of Example 1, the results are:

$$
\lambda_{P N}^{\sigma(1)}=14 u, \quad \lambda_{P N}^{\sigma(2)}=18 u, \quad \lambda_{P N}^{\sigma(3)}=20 u, \quad \lambda_{P N}^{\sigma(4)}=21 u, \quad \lambda_{P N}^{\sigma(5)}=23 u, \quad \lambda_{P N}^{\sigma(6)}=24 u, \quad \lambda_{P N}^{\sigma(7)}=30 u, \quad \lambda_{P N}^{\sigma(8)}=40 u .
$$

Meantime, the corresponding weight information is:

$$
w_{P N}^{\sigma(1)}=0.05, \quad w_{P N}^{\sigma(2)}=0.05, w_{P N}^{\sigma(3)}=0.3, w_{P N}^{\sigma(4)}=0.1, \quad w_{P N}^{\sigma(5)}=0.1, \quad w_{P N}^{\sigma(6)}=0.3, \quad w_{P N}^{\sigma(7)}=0.05, \quad w_{P N}^{\sigma(8)}=0.05
$$

Under a certain value of $\varepsilon$, we utilize (4)-(8) to calculate the composite multiplicative index for each evaluation outcome. Then, we obtain the granulation result by comparing the composite multiplicative index. The granulation procedure of the evaluation results of $\lambda_{P N}$ is shown in Fig. 7.

From Fig. 7, we analyze four cases, i.e., $\varepsilon=0.03, \varepsilon=0.5, \varepsilon=1$ and $\varepsilon=4$. We display the interval granulation procedure of $\lambda_{P N}$ with different values of $\varepsilon$, respectively. As the sketch presented in Fig. 2, we can find the maximization of the composite multiplicative index toward the left of $m_{P N}$ and determine the lower bound of interval, e.g., the result of Example 1. To the right of $m_{P N}$, we also can determine the upper bound of the interval. Under the different values of $\varepsilon$, we can adopt the similar way to obtain the corresponding information granules. Following the ascending order of $\varepsilon$, the information granulation results are: [18u, 24u], [20u, 24u], [20u, 24u] and [21u, 23u]. With the decreasing of $\varepsilon$, the composite multiplicative index of each evaluation is enlarged. The distance between the lower and upper bounds of the information granule of loss function has the trend of expansion with the decreasing of $\varepsilon$. It verifies the result presented in Proposition 2.

Following the results of Pedrycz and Homenda [37], the value of $\varepsilon$ is given. In this example, suppose the value of $\varepsilon$ is 0.5 . With the aid of the principle of justifiable granularity, we obtain the interval information granules of loss functions as follows: $\lambda_{P P}=[0,2 u], \lambda_{B P}=[10 u, 15 u], \lambda_{N P}=[23 u, 28 u], \lambda_{P N}=[20 u, 24 u], \lambda_{B N}=[10 u, 12 u]$, $\lambda_{N N}=[0.8 u, 2 u]$. 
Table 8: The granulation results of loss functions with different values of $\varepsilon$ ( $u$ is a unit cost)

\begin{tabular}{|c|c|c|c|c|c|c|}
\hline$\varepsilon$ & $\lambda_{P P}$ & $\lambda_{B P}$ & $\lambda_{N P}$ & $\lambda_{P N}$ & $\lambda_{B N}$ & $\lambda_{N N}$ \\
\hline 0 & {$[0,2.5 u]$} & {$[4 u, 20 u]$} & {$[8 u, 41 u]$} & {$[14 u, 40 u]$} & {$[8.5 u, 18 u]$} & {$[0,3 u]$} \\
0.005 & {$[0,2.5 u]$} & {$[4 u, 20 u]$} & {$[8 u, 41 u]$} & {$[14 u, 40 u]$} & {$[8.5 u, 18 u]$} & {$[0,3 u]$} \\
0.03 & {$[0,2.5 u]$} & {$[9 u, 20 u]$} & {$[14 u, 41 u]$} & {$[18 u, 24 u]$} & {$[8.5 u, 18 u]$} & {$[0,3 u]$} \\
0.1 & {$[0,2.5 u]$} & {$[9 u, 20 u]$} & {$[20 u, 34 u]$} & {$[20 u, 24 u]$} & {$[8.5 u, 18 u]$} & {$[0,3 u]$} \\
0.3 & {$[0,2.5 u]$} & {$[10 u, 15 u]$} & {$[21 u, 28 u]$} & {$[20 u, 24 u]$} & {$[9 u, 12 u]$} & {$[0,3 u]$} \\
0.5 & {$[0,2 u]$} & {$[10 u, 15 u]$} & {$[23 u, 28 u]$} & {$[20 u, 24 u]$} & {$[10 u, 12 u]$} & {$[0.8 u, 2 u]$} \\
0.6 & {$[0,2 u]$} & {$[10 u, 15 u]$} & {$[23 u, 28 u]$} & {$[20 u, 24 u]$} & {$[10 u, 12 u]$} & {$[0.8 u, 2 u]$} \\
0.85 & {$[0,1.05 u]$} & {$[10 u, 15 u]$} & {$[23 u, 28 u]$} & {$[20 u, 24 u]$} & {$[10 u, 12 u]$} & {$[u, 2 u]$} \\
1 & {$[0,1.05 u]$} & {$[10 u, 15 u]$} & {$[23 u, 28 u]$} & {$[20 u, 24 u]$} & {$[10 u, 12 u]$} & {$[u, 2 u]$} \\
4 & {$[0,1.05 u]$} & {$[13.25 u, 14.2 u]$} & {$[26.75 u, 28 u]$} & {$[21 u, 23 u]$} & {$[11 u, 12 u]$} & {$[1.5 u, 2 u]$} \\
\hline
\end{tabular}

Step 4: For the interval information granules of loss functions, the decision makers also accept the distance between the lower and upper bound of each loss function when $\varepsilon$ is 0.5 .

Step 5: Given the value of $\theta$ is 0.5 [22], we further transform the interval-valued information granule and determine the values of loss functions by using (11). The calculated results are: $m_{0.5}\left(\lambda_{P P}\right)=1 u, m_{0.5}\left(\lambda_{B P}\right)=12.5 u, m_{0.5}\left(\lambda_{N P}\right)=$ $25.5 u, m_{0.5}\left(\lambda_{P N}\right)=22 u, m_{0.5}\left(\lambda_{B N}\right)=11 u$ and $m_{0.5}\left(\lambda_{N N}\right)=1.4 u$.

Step 6: Based on (10), we compute the thresholds values: $\alpha=0.4889, \beta=0.4248$ and $\gamma=0.4568$.

Step 7: Through comparing (11) and (12), we confirm the corresponding judgement criterion (P1)-(N1). Thus, the decision rules of the supplier $x$ are obtained:

$$
\begin{aligned}
& \text { (P1) If } \operatorname{Pr}(C \mid[x]) \geq 0.4889 \text {, decide } x \in \operatorname{POS}(C) \text {; } \\
& \text { (B1) If } 0.4248<\operatorname{Pr}(C \mid[x])<0.4889 \text {, decide } x \in \operatorname{BND}(C) \text {; } \\
& \text { (N1) If } \operatorname{Pr}(C \mid[x]) \leq 0.4248 \text {, decide } x \in \operatorname{NEG}(C) .
\end{aligned}
$$

For the supplier $x$, if the conditional probability $\operatorname{Pr}(C \mid[x])$ is greater than or equal to 0.4889 , we select the supplier. If the conditional probability $\operatorname{Pr}(C \mid[x])$ is less than or equal to 0.4248 , we directly reject the supplier. If the conditional probability $\operatorname{Pr}(C \mid[x])$ is between 0.4248 and 0.4889 , we defer our decision and need to further collect some additional information.

During the decision analysis of GDM-based three-way decisions, it involves many parameters. In what follows, we successively analyze the influences of the parameters to the strategic supplier selection in details.

(1) The impact of $\varepsilon$ to the information granulations of loss functions

The essential role of $\varepsilon$ is to calibrate an impact of the specificity criterion on the constructed information granule [37]. Based on the evaluations of Table 7, we discuss the impact of $\varepsilon$ to the information granulations of loss functions. With respect to different values of $\varepsilon$, the granulation results of loss functions are shown in Table 8 .

From Table 8, the distance between the lower and upper bounds of the interval presented in Table 8 has the trend of shrink with the increasing of $\varepsilon$. For clarity, we describe the granulation results of loss functions reported in Table 8, see Fig. 8.

In Fig. 8, the interval outcomes of each loss function under different values of $\varepsilon$ are described. The distance between the lower and upper bounds of the information granule of loss function is amplified or invariant with the decreasing of $\varepsilon$. The conclusion supports the result presented in Proposition 3 .

(2) The transform outcomes of loss functions and the thresholds values with different values of $\varepsilon$ and $\theta$

Based on (9) and (10), the transform outcomes of loss functions and the thresholds with different values of $\varepsilon$ and $\theta$ are further discussed in Fig. 9.

With regard to the results of Fig. 9, we find that the transform outcomes of loss functions are uncertain with the increasing of $\varepsilon$, when the value of $\theta$ is constant. Besides, the transform outcomes of loss functions are increasing with the increasing of $\theta$, when the value of $\varepsilon$ is constant. 

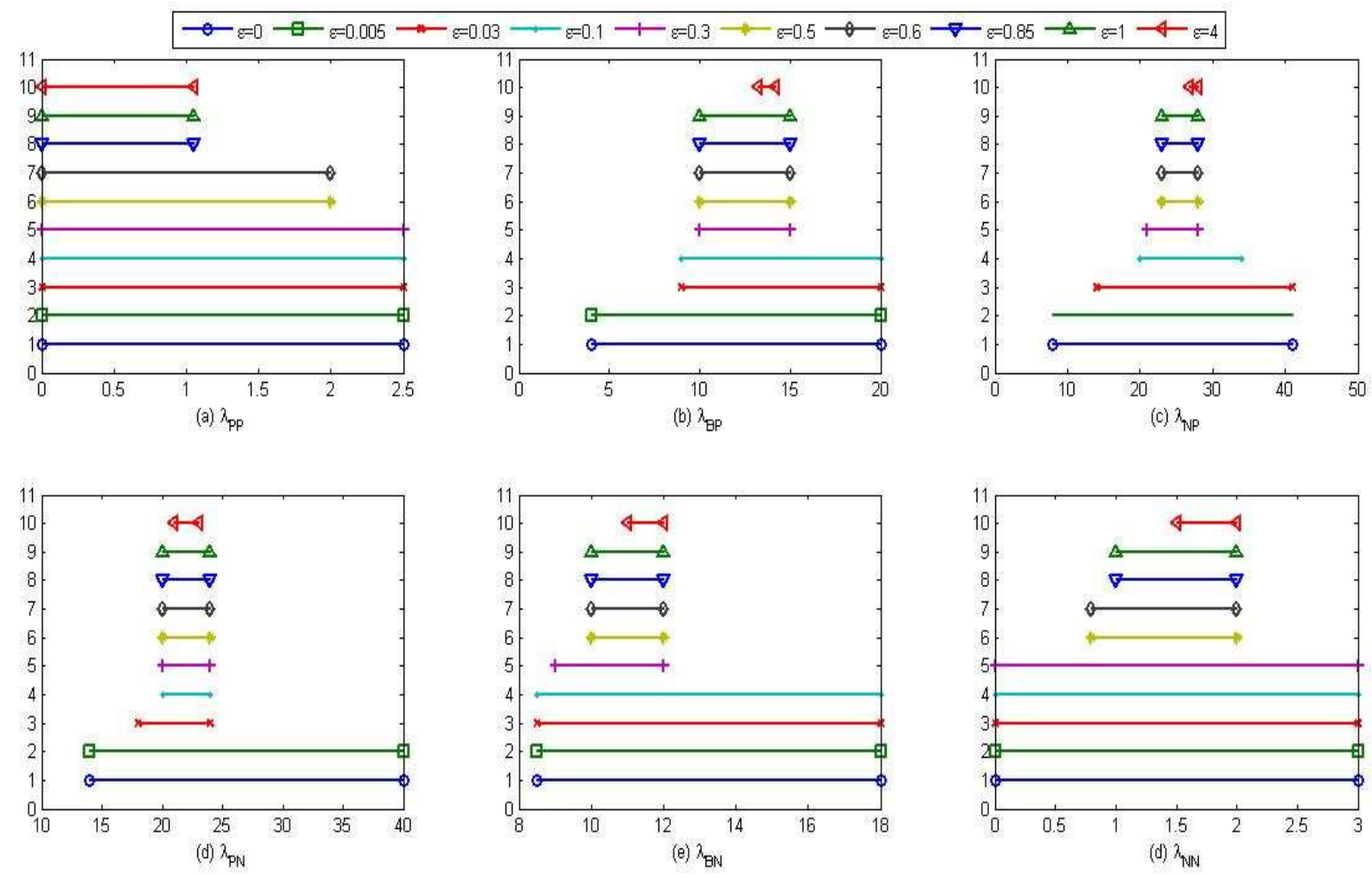

Figure 8: The granulation results of loss functions with different values of $\varepsilon$ ( $u$ is a unit cost)
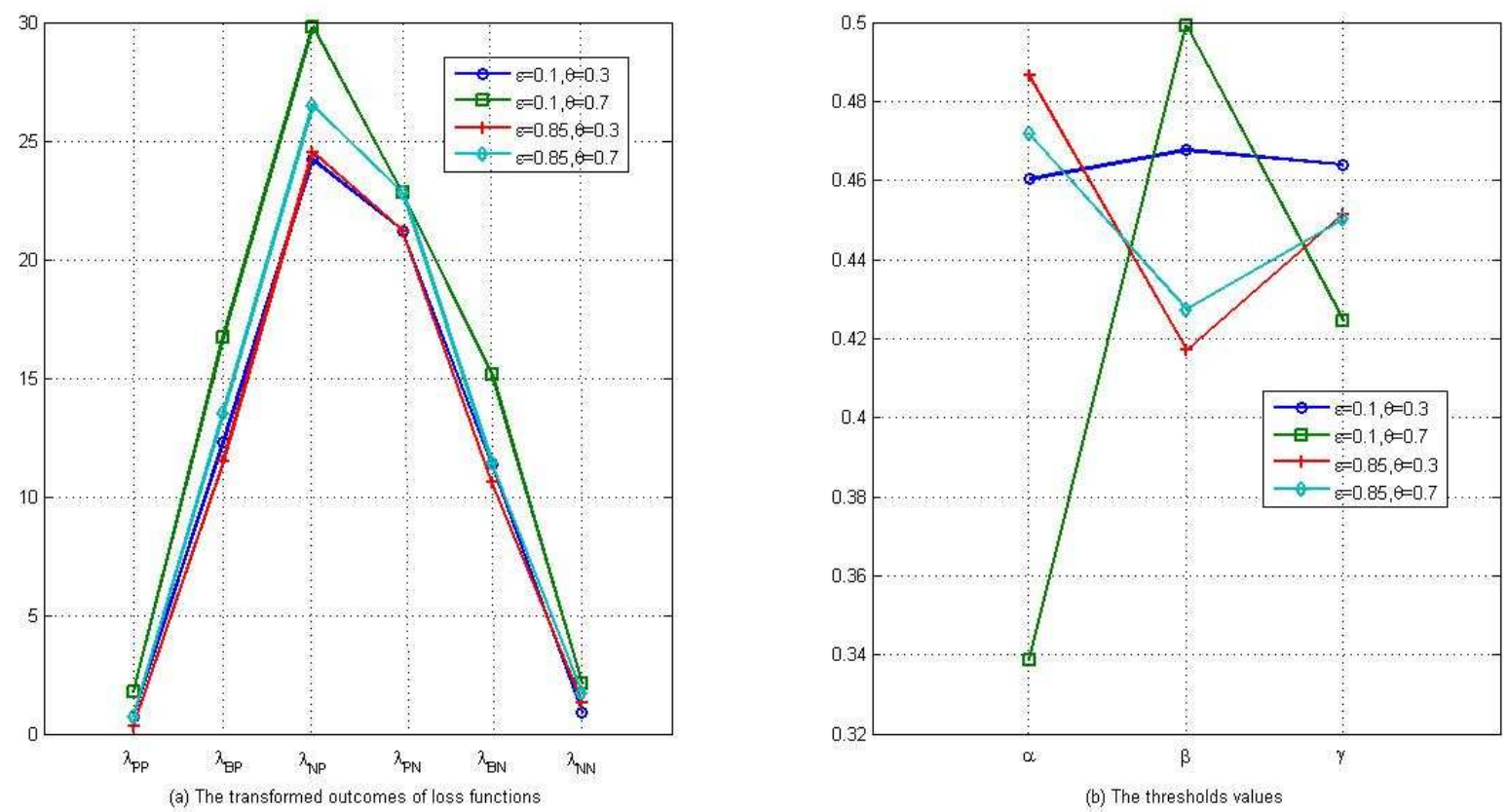

(b) The thresholds values

Figure 9: The transform outcomes of loss functions and the thresholds values with different values of $\varepsilon$ and $\theta$ ( $u$ is a unit cost) 

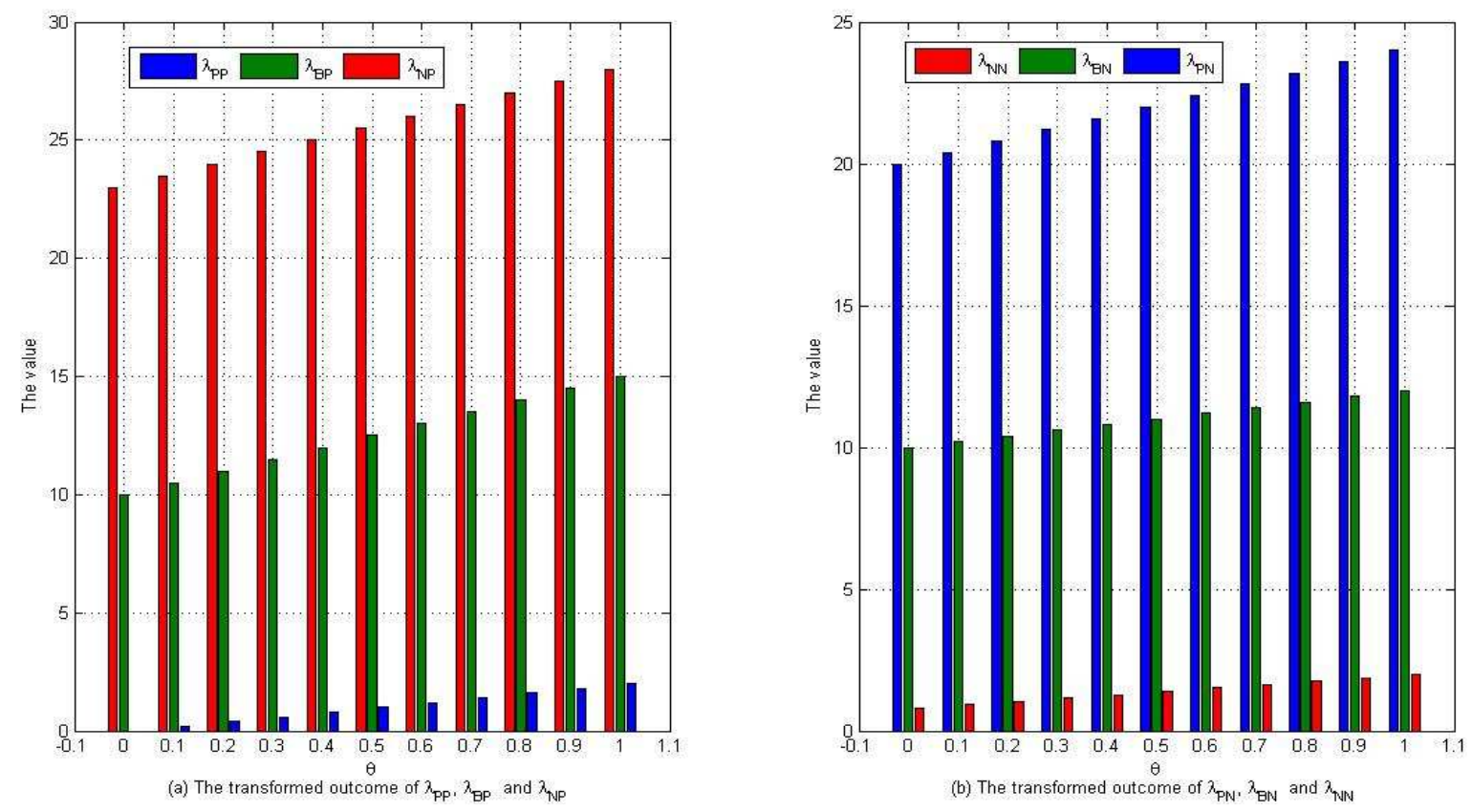

Figure 10: The transform outcomes of loss functions with different values of $\theta$ when $\varepsilon$ is 0.5

For the thresholds values, they do not keep the same pace with the variations of $\varepsilon$ and $\theta$. We assume that $\varepsilon$ is 0.5 . In this case, the interval-valued loss functions are generated as follows: $\lambda_{P P}=[0,2 u], \lambda_{B P}=[10 u, 15 u]$, $\lambda_{N P}=[23 u, 28 u], \lambda_{P N}=[20 u, 24 u], \lambda_{B N}=[10 u, 12 u], \lambda_{N N}=[0.8 u, 2 u]$. According to (9), we calculate the transform outcomes of each loss function with different values of $\theta$. The results are shown in Fig. 10.

In light of the results presented in Fig. 10, the relationships among the transform outcomes of the loss functions are consistent with (1)-(2). By using (10), the thresholds values $(\alpha, \beta, \gamma)$ with different values of $\theta$ are further calculated. The results are shown in Fig. 11.

From Fig. 11, the relationship among the thresholds is: $0 \leq \beta<\gamma<\alpha \leq 1$. With the increasing of $\theta$, the distance between $\alpha$ and $\beta$ inclines to shrink.

(3) The decision rules with different values of $\theta$ when $\varepsilon$ is constant

Continuing the discussion of Fig. 11, we deduce the decision rules with different values of $\theta$. On the basis of the condition $0 \leq \beta<\gamma<\alpha \leq 1$, we generate decision rules (P1)-(N1), i.e., $\operatorname{POS}(C), \mathrm{BND}(C)$ and $\mathrm{NEG}(C)$. With respect to $(\mathrm{P} 1)-(\mathrm{N} 1)$, the three decision regions rely on the thresholds $(\alpha, \beta)$. Hence, the decision rules with different values of $\theta$ are described in Fig. 12.

In Fig. 12, we use three ranges to individually represent decision rules $\operatorname{POS}(C), \mathrm{BND}(C)$ and $\mathrm{NEG}(C)$. With the increasing of $\theta$, the region $\operatorname{BND}(C)$ is inclined to get smaller and smaller. It implies that the deferment decision $\operatorname{BND}(C)$ is becoming more and more certain and transfers into the other regions, e.g., the acceptance $\operatorname{POS}(C)$ or the rejection $\operatorname{NEG}(C)$.

In general, we adopt GDM-based three-way decisions to solve the strategic supplier selection problem. In this example, we elaborate its decision procedure. We also discuss the influences of the parameters to the strategic supplier selection. The results successfully verify the results presented in Propositions 1-4.

\section{Experimental analysis}

Under the background of GDM, the inconsistency of experts reflects the effectiveness of GDM [20, 43]. The distance method proposed by $\mathrm{Xu}$ [43] is widely used to calculate the inconsistency. Its primary method is the average deviation method. In Section 4.4, GDM-based three-way decisions are proposed by using granular computing, which 


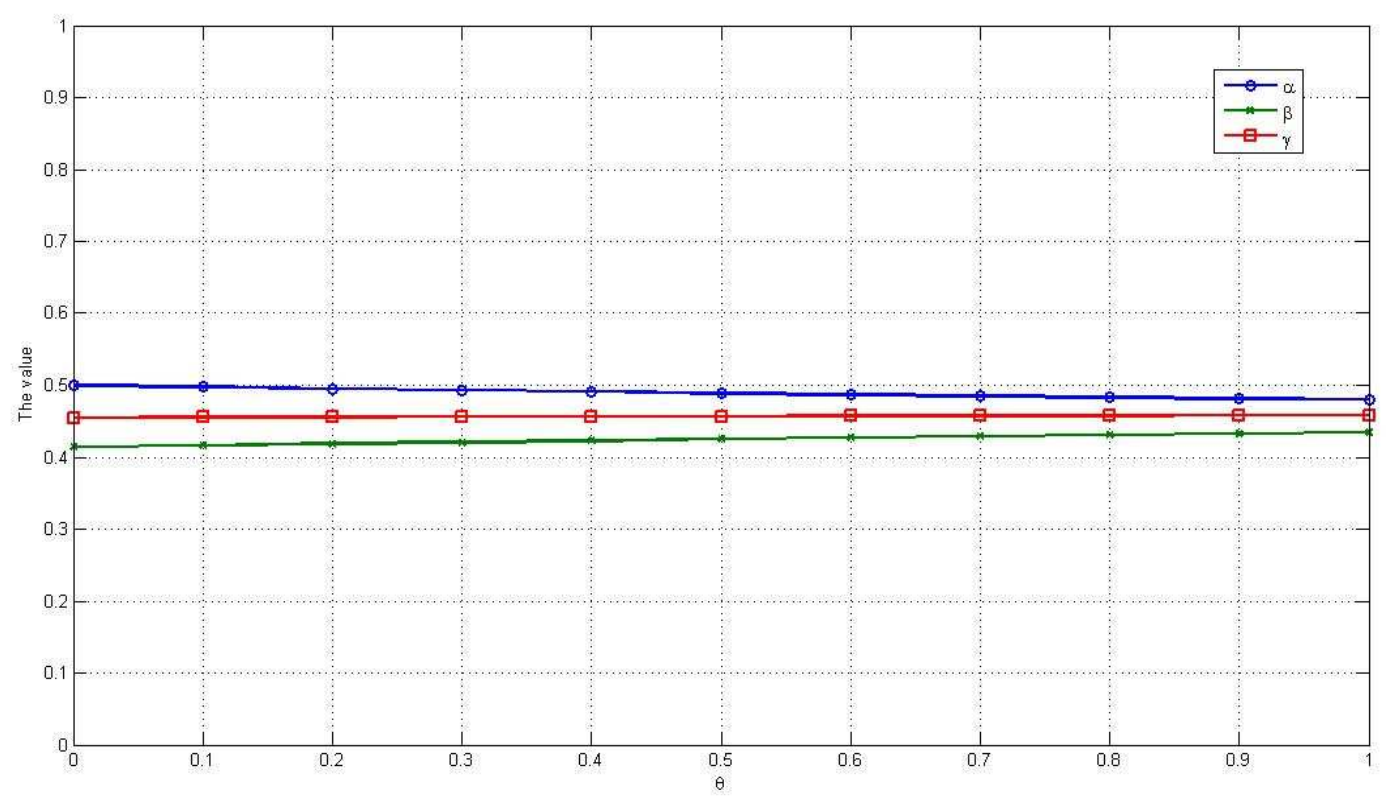

Figure 11: The thresholds values with different values of $\theta$ when $\varepsilon$ is 0.5

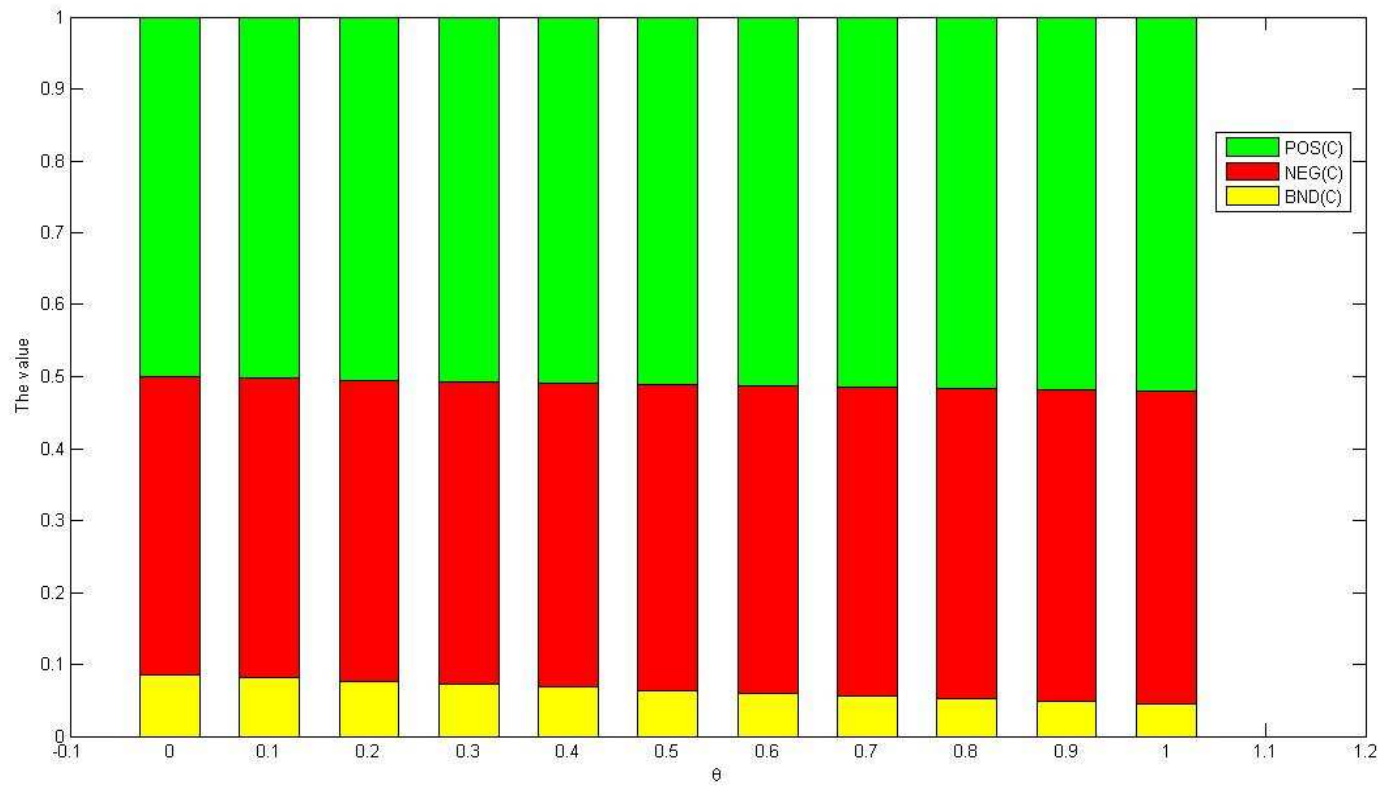

Figure 12: The decision rules with different values of $\theta$ when $\varepsilon$ is 0.5 
utilize the information granulation and degranulation strategies. In this section, we compare the performance of our proposed method with the average deviation method of Xu [43] by experimental analysis. In light of the results reported in $[21,51,59]$, we use the error rate to evaluate the performance as follows:

$$
e=\frac{n_{C \rightarrow \mathrm{NEG}(C)}+n_{\neg C \rightarrow \mathrm{POS}(C)}}{Q} \times 100 \% .
$$

where $Q$ is the total number of objects presented in the universe, $n_{C \rightarrow \mathrm{NEG}(C)}$ is the number of objects belonging to $C$ that are classified as belonging to $\mathrm{NEG}(C), n_{\neg C \rightarrow \operatorname{POS}(C)}$ is the number of objects belonging to $\neg C$ that are classified as belonging to $\operatorname{POS}(C)$. The smaller of error rate $e$ implies that the performance is the better.

According to the results of [21], we conduct a series of experiments to compare the performances of three-way decision using the different methods. On basis of [21], we select four data sets from the machine learning data repository, University of California at Irvine, where the basic information of data sets is outlined in Table 9 [21].

Table 9: A description of data sets [21]

\begin{tabular}{llllll}
\hline No. & Data sets & Abbreviation & Samples & Attributes & Classes \\
\hline 1 & Chess (King-Rook vs. King-Pawn) & Chess & 3196 & 36 & 2 \\
2 & Monk's Problems & Monk & 432 & 6 & 2 \\
3 & Nursery & Nursery & 12960 & 8 & 5 \\
4 & Tic-Tac-Toe Endgame & Tic-Tac-Toe & 958 & 9 & 2 \\
\hline
\end{tabular}

Before the experimental analysis, date sets need to be preprocessed. Based on the results of [21], some data sets are consistent information systems. In order to exert the advantage of three-way decisions [51], we delete some attributes of data sets in advance. In this paper, we discuss two states $\Omega=\{C, \neg C\}$. The classes of some data sets of Table 9 are more than 2 . Therefore, we need merge some classes and definite the concept of $C$. The pretreatment of these data sets are shown in Table 10.

Table 10: The pretreatment of data sets [21]

\begin{tabular}{lll}
\hline Data sets & Definition of the concept $C$ & Deleted Attributes \\
\hline Chess & $\{$ won $\}$ & The 15th attribute \\
Monk & $\{1\}$ & $\{\mathrm{a} 1\}$ \\
Nursery & \{priority, spec_prior & \{has_nurs \\
Tic-Tac-Toe & \{positive & \{bottom-left-square, bottom-middle-square, bottom-right-square \\
\hline
\end{tabular}

For each data set, we randomly divide it into 5 equal portions [21]. Based on these portions, we successively accumulate them and form 5 samples, namely, 1-5. In the section, we focus on the comparison between our proposed method with the average deviation method. For convenience, we assume the loss functions evaluated by experts are shown in Table 7. In this scenario, the weighted means of loss functions are calculated as: $m_{P P}=0.88 u, m_{B P}=$ $13.25 u, m_{N P}=26.75 u, m_{P N}=22.7 u, m_{B N}=11.91 u$ and $m_{N N}=1.635 u$. Following the discussion of Section 5, the set of experts is $E=\left\{e_{1}, e_{2}, e_{3}, e_{4}, e_{5}, e_{6}, e_{7}, e_{8}, e_{9}, e_{10}, e_{11}\right\}$ and the corresponding weight vector of experts is $W=\{0.05,0.05,0.05,0.05,0.05,0.05,0.1,0.1,0.1,0.2,0.2\}^{T}$.

Under the average deviation method, three-way decisions are deduced based on the weighted means of loss functions [43]. However, with the aid of our proposed method, it mainly includes two key steps. One is the information granulation of the determination of loss functions, i.e., (4)-(8). The other is the information degranulation of intervalvalued loss function based on the transformed formula (9). In light of the results of Table 8, we directly excerpt the interval information granules of loss functions when the value of $\varepsilon$ is 0.005 . The results are: $\lambda_{P P}=[0,2.5 u]$, $\lambda_{B P}=[4 u, 20 u], \lambda_{N P}=[8 u, 41 u], \lambda_{P N}=[14 u, 40 u], \lambda_{B N}=[8.5 u, 18 u], \lambda_{N N}=[0,3 u]$. Then, we adopt the information degranulation of (9) to determine the loss functions with a certain of $\theta$. Based on the sample and corresponding 

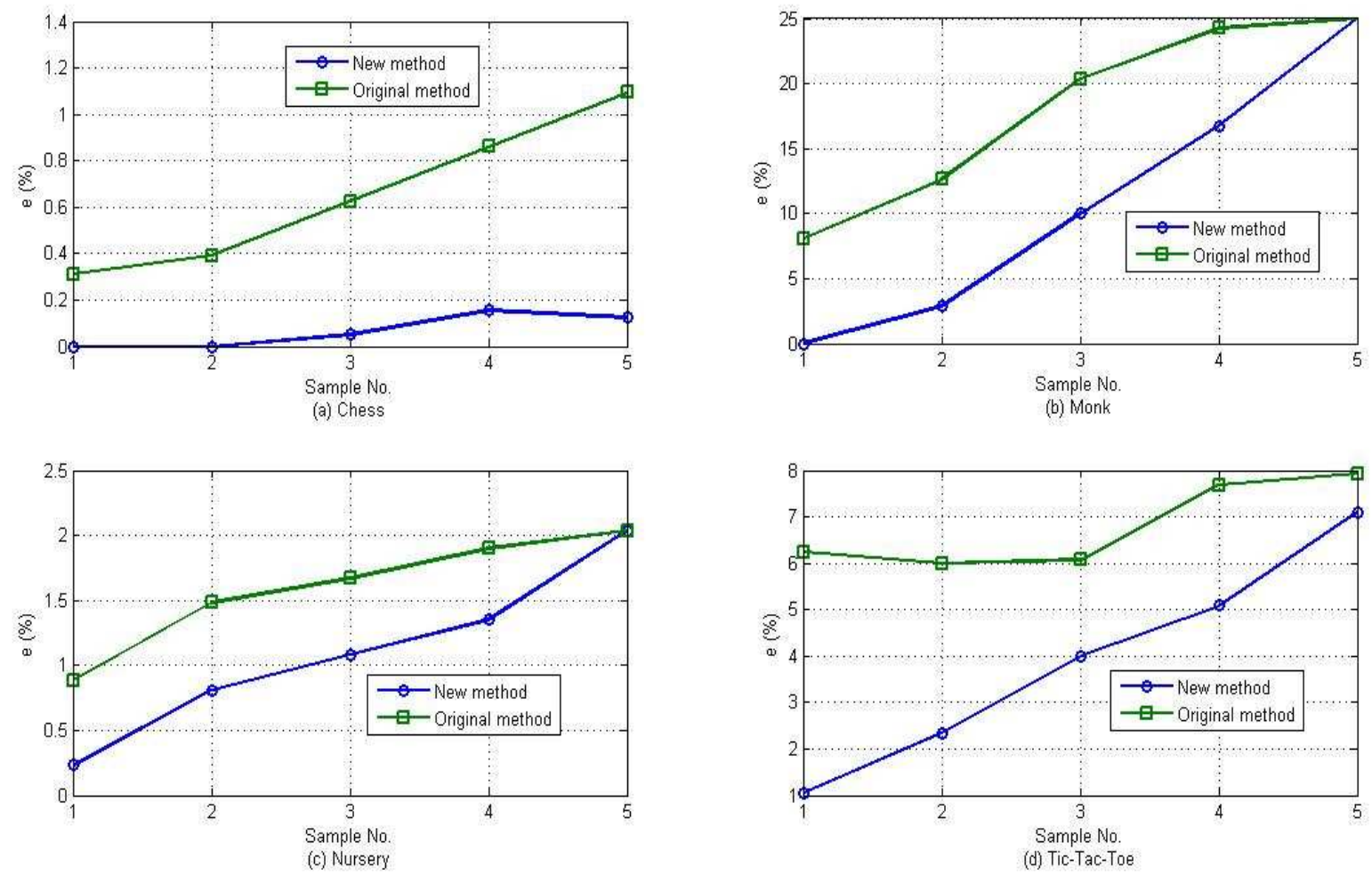

Figure 13: The comparison between our proposed methods and the average deviation method

loss functions, we calculate the error rates of our proposed methods and the average deviation method, respectively. The results are elaborated in Fig. 13.

In Fig. 13, the new method denotes our proposed method. The original method corresponds to the average deviation method. We find that the error rate of the new method is not more than the results of the original method in the each sample. It implies that the performance of our proposed method is better than the average deviation method. The comparison analysis validates the performance of our proposed method. It also reflects the tolerance of interval-valued information granule.

\section{Conclusions}

Based on three-way decisions with DTRSs model, we consider the GDM evaluation mode to determine the loss functions. In this paper, we propose GDM-based three-way decisions with the aid of the principle of justifiable granularity and the interval comparison method. The proposed method utilizes the information granulation and degranulation strategies. The novel three-way decisions successfully solve the information aggregations of multiple experts. In additions, GDM provides a new semantic interpretation for the loss functions of three-way decisions and interprets the determination of the imprecise loss function presented in [20, 21, 22]. Yao and Herbert [46] utilized game theory to automatically determine the parameters. In order to automatically get the loss functions of GDM, we can draw lessons from the results of $[2,46]$ and extend our proposed model. It is worth noting that the value of $\varepsilon$ also resorts to the result of information granulation in practice. With the help of machine learning methods, we will further discuss the adjustment of $\varepsilon$ in our future work.

\section{Acknowledgements}

This work is partially supported by the National Science Foundation of China (Nos. 71401026, 71432003, 71571148, 71201133, 71490722), the Fundamental Research Funds for the Central Universities of China (Nos. 
ZYGX2014J100, SWJTU12CX117) and the Social Science Planning Project of the Sichuan Province (No. SC15C009).

\section{References}

[1] C. Araz, I. Ozkarahan, Supplier evaluation and management system for strategic sourcing based on a new multicriteria sorting procedure, International Journal of Production Economics 106 (2007) 585-606.

[2] N. Azam, J.T. Yao, Interpretation of equilibria in game-theoretic rough sets, Information Sciences 295 (2015) 586-599.

[3] G. Büyüközkan, O. Feyzioğlu, E. Nebol, Selection of the strategic alliance parter in logisitics value chain, International Journal of Production Economics 113 (2008) 148-158.

[4] F.J. Cabrerizo, E. Herrera-Viedma, W. Pedrycz, A method based on PSO and granular computing of linguistic information to solve group decision making problems defined in heterogeneous contexts, European Journal of Operational Research 230 (3) (2013) 624-633.

[5] L. De Boer, E. Labro, P. Morlacchi, A review of methods supporting supplier selection, European Journal of Purchasing and Supply Management 7 (2) (2001) 75-89.

[6] Z. Degraeve, E. Labro, F. Roodhooft, An evaluation of supplier selection methods from a total cost of ownership perspective, European Journal of Operational Research 125 (1) (2000) 34-58.

[7] X.F. Deng, Y.Y. Yao, Decision-theoretic three-way approximations of fuzzy sets, Information Sciences 279 (2014) 702-715.

[8] R.O. Duda, P.E. Hart, Pattern classification and scene analysis, Wiley Press, New York, 1973.

[9] R. Florez-Lopez, Strategic supplier selection in the added-value perspective: A CI approach, Information Sciences 177 (2007) 1169-1179.

[10] S.H. Ghodsypour, C. O'Brien, The total cost of logistics in supplier selection, under conditions of multiple sourcing, multiple criteria and capacity constraint, International Journal of Production Economics 73 (1) (2001) 15-27.

[11] K. Goffin, F. Lemke, M. Szwejczewski, An exploratory study of 'close' supplier-manufacturer relationships, Journal of Operations Management 24 (2) (2006) 189-209.

[12] S. Greco, R. Slowinski, Y.Y. Yao, Bayesian decision theory for dominance-based rough set approach, in: J.T. Yao et al.(Eds): RSKT2007, LNAI 4481, Springer, Berlin, 2007, pp. 134-141.

[13] W. Ho, X. Xu, P.K. Dey, Multi-criteria decision making approaches for supplier evaluation and selection: a literature review, European Journal of Operational Research 202 (1) (2010) 16-24.

[14] B.Q. Hu, Three-way decisions space and three-way decisions, Information Sciences 281 (2014) 21-52.

[15] X.Y. Jia, W.H. Liao, Z.M. Tang, L. Shang, Minimum cost attribute reduction in decision-theoretic rough set models, Information Sciences 219 (2013) 151-167.

[16] Y. Li, C. Zhang, J.R. Swan, An information filtering model on the web and its application in JobAgent, Knowledge-Based Systems 13 (2000) 285-296.

[17] W. Li, D.Q. Miao, W.L. Wang, N. Zhang, Hierarchical rough decision theoretic framework for text classification, in: Proceedings of the 9th IEEE International Conference on Cognitive Informatics, 2010, pp. 484-489.

[18] H.X. Li, X.Z. Zhou, Risk decision making based on decision-theoretic rough set: a three-way view decision model, International Journal of Computational Intelligence Systems 4 (2011) 1-11.

[19] W.T. Li, W.H. Xu, Double-quantitative decision-theoretic rough set, Information Sciences 316 (2015) 54-67.

[20] D.C. Liang, D. Liu, W. Pedrycz, P. Hu, Triangular fuzzy decision-theoretic rough sets, International Journal of Approximate Reasoning 54 (8) (2013) 1087-1106.

[21] D.C. Liang, D. Liu, Systematic studies on three-way decisions with interval-valued decision-theoretic rough sets, Information Sciences 276 (2014) 186-203.

[22] D.C. Liang, D. Liu, A novel risk decision making based on decision-theoretic rough sets under hesitant fuzzy information, IEEE Transaction on Fuzzy Systems 23(2) (2015) 237-247.

[23] D.C. Liang, D. Liu, Deriving three-way decisions from intuitionistic fuzzy decision-theoretic rough sets, Information Sciences 300 (2015) $28-48$.

[24] D.C. Liang, W. Pedrycz, D. Liu, P. Hu, Three-way decisions based on decision-theoretic rough sets under linguistic assessment with the aid of group decision making, Applied Soft Computing 29 (2015) 256-269.

[25] P. Lingras, M. Chen, D.Q. Miao, Rough cluster quality index based on decision theory, IEEE Transactions on Knowledge and Data Engineering 21 (7) (2009) 1014-1026.

[26] J.S. Liu, X.Z. Wang, B.Y. Zhang, The ranking of interval numbers, Journal of Engineering Mathematics 18 (4) (2001) 103-109. (in chinese)

[27] D. Liu, Y.Y. Yao, T.R. Li, Three-way investment decisions with decision-theoretic rough sets, International Journal of Computational Intelligence Systems 4 (2011) 66-74.

[28] D. Liu, T.R. Li, D. Ruan, Probabilistic model criteria with decision-theoretic rough sets, Information Sciences 181 (2011) $3709-3722$.

[29] D. Liu, T.R. Li, D.C. Liang, Three-way government decision analysis with decision-theoretic rough sets, International Journal of Uncertainty, Fuzziness and Knowledge-Based Systems 20 (2012) (Supp. 1) 119-132.

[30] D. Liu, T.R. Li, H.X. Li, Interval-valued decision-theoretic rough sets, Computer Science 39 (7) (2012) 178-214 (in Chinese).

[31] D. Liu, T.R. Liu, D.C. Liang, Incorporating logistic regression to decision-theoretic rough sets for classifications, International Journal of Approximate Reasoning 55 (2014) 197-210.

[32] D. Liu, D.C. Liang, C.C. Wang, A novel three-way decision model based on incomplete information system, Knowledge-Based Systems, doi: 10.1016/j.knosys.2015.07.036.

[33] X.A. Ma, G.Y. Wang, H. Yu, T.R. Li, Decision region distribution preservation reduction in decision-theoretic rough set model, Information Sciences 278 (2014) 614-640.

[34] Z. Pawlak, Rough sets, International Journal of Computer and Information Science 11 (1982) 341-356.

[35] W. Pedrycz, M.L. Song, Analytic hierarchy process (AHP) in group decision making and its optimization with an allocation of information granularity, IEEE Transactions on Fuzzy Systems 19 (3) (2011) 527-539. 
[36] W. Pedrycz, Granular Computing: Analysis and Design of Intelligent Systems, CRC Press/Francis Taylor, Boca Raton, 2013.

[37] W. Pedrycz, W. Homenda, Building the fundamentals of granular computing: A principle of justifiable granularity, Applied Soft Computing 13 (2013) 4209-4218.

[38] W. Pedrycz, Allocation of information granularity in optimization and decision-making models: Towards building the foundations of Granular Computing, European Journal of Operational Research 232(1) (2014) 137-145.

[39] Y.H. Qian, H. Zhang, Y.L. Sang, J.Y. Liang, Multigranulation decision-theoretic rough sets, International Journal of Approximate Reasoning 55 (2014) 225-237.

[40] B.Z. Sun, W.M. Ma, H.Y. Zhao, Decision-theoretic rough fuzzy set model and application, Information Sciences 283 (2014) 180-196.

[41] B.Z. Sun, W.M. Ma, Rough approximation of a preference relation by multi-decision dominance for a multi-agent conflict analysis problem, Information Sciences 315 (2015) 39-53.

[42] L.Z. Wang, X.D. Liu, W. Pedrycz, Effective intervals determined by information granules to improve forecasting in fuzzy time series, Expert Systems with Applications, 40(14) (2013) 5673-5679.

[43] Z.S. Xu, Au automatic approach to reaching consensus in multiple attribute group decision making, Computer \& Industrial Engineering 56 (2009) 1369-1374.

[44] X.P. Yang, J.T. Yao, Modelling multi-agen three-way decisions with decision-theoretic rough sets, Fundamenta Informaticae 115(2-3) (2012) 157-171.

[45] J.T. Yao, J.P. Herbert, Web-based support systems with rough set analysis, in: M. Kryszkiewicz et al. (Eds.): RSEIP 2007, LNAI 4585, Springer, Berlin, 2007, pp. 360-370.

[46] J.T. Yao, J.P. Herbert, A game-theoretic perspective on rough set analysis, Journal of Chongqing University of Posts and Telecommunications (Natural Science Edition) 20 (3) (2008) 291-298.

[47] J.T. Yao, N. Azam, Web-based medical decision support systems for three-way medical decision making with game-theoretic rough sets, IEEE Transactions on Fuzzy Systems 23(1) (2015) 3-15.

[48] Y.Y. Yao, S.K.M. Wong, P. Lingras, A decision-theoretic rough set model, in: Z. W. Ras, M. Zemankova, M. L. Emrich (Eds.): Methodologies for Intelligent Systems, 5, New York: North-Holland, 1990, pp. 17-24.

[49] Y.Y. Yao, S.K.M. Wong, A decision theoretic framework for approximating concepts, International Journal of Man-machine Studies 37 (1992) 793-809.

[50] Y.Y. Yao, Three-way decisions with probabilistic rough sets, Information Sciences 180 (2010) 341-353.

[51] Y.Y. Yao, The superiority of three-way decision in probabilistic rough set models, Information Sciences 181(6) (2011) 1080-1096.

[52] H. Yu, Z.G. Liu, G.Y. Wang, An automatic method to determine the number of clusters using decision-theoretic rough set, International Journal of Approximate Reasoning 55 (2014) 142-155.

[53] Y.Y. Yao, B. Zhou, Two bayesian approaches to rough sets, European Journal of Operational Research, 2015, doi: 10.1016/j.ejor.2015.08.053.

[54] H. Yu, C. Zhang, G.Y. Wang, A tree-based incremental overlapping clustering method using the three-way decision theory, Knowledge-Based Systems, doi: 10.1016/j.knosys.2015.05.028.

[55] X.Y. Zhang, D.Q. Miao, Reduction target structure-based hierarchical attribute reduction for two-category decision-theoretic rough sets, Information Sciences 277 (2014) 755-776.

[56] H.R. Zhang, F. Min, Three-way recommender systems based on random forests, Knowledge-Based Systems, doi: 10.1016/j.knosys.2015.06.019.

[57] X.R. Zhao, B.Q. Hu, Fuzzy and interval-valued fuzzy decision-theoretic rough set approaches based on fuzzy probability measure, Information Sciences 298 (2015) 534-554

[58] B. Zhou, Multi-class decision-theoretic rough sets, International Journal of Approximate Reasoning 55 (2014) 211-224.

[59] B. Zhou, Y.Y. Yao, J.G. Luo, Cost-sensitive three-way email spam filtering, Journal of Intelligent Information Systems 42(1) (2014) 19-45 